\title{
HOW TO COMPENSATE A SPREADING DISTURBANCE FOR A CLASS OF NONLINEAR SYSTEMS
}

\author{
Youssef QARAAI*，Abdes Samed BeRNOUSSI*, Abdelhaq El JAI** \\ * MPPRN Laboratory, B.P. 416, FST of Tangier, Morocco \\ e-mail: \{qaraai youssef, bersamed16\}@yahoo.fr \\ ** LAMPS/LTS, University of Perpignan 66860, France \\ e-mail: aej@univ-perp.fr
}

\begin{abstract}
We consider a system which is assumed to be affected by an expanding disturbance which occurs at the initial time. The compensation of the disturbance is accomplished by extending the concept of remediability to a class of nonlinear systems. The results are implemented and illustrated with a nonlinear distributed model.
\end{abstract}

Keywords: distributed nonlinear systems, disturbance, spreadability, remediability, control.

\section{Introduction}

Systems analysis concerns a set of concepts which lead to a better understanding of systems and their evolution. This allows more convenient controls to achieve any objective. In the case of distributed parameter systems, in which the space variable plays an important role, there is a wide literature on controllability, observability, stability, detectability, compensation, etc., see (Afifi et al., 2002; Bernoussi and El Jai, 2000; Bernoussi and Amharref, 2003; El Jai and Kassara, 1994; El Jai et al., 1995; El Jai, 2002; El Jai, 2004; Kassara and El Jai, 1983) and the references therein. Moreover, the analysis itself has to deal not with the whole domain, but with its specific subdomains of interest. Thus, since the beginning of the 1990s, motivated by practical applications, Afifi, El Jai, and Zerrik (2008) have introduced and studied the so-called regional analysis. Generally speaking, such analysis aims to analyze or control a system in which an objective function is defined only on a prescribed subregion. Therefore the system dynamics are defined in the whole of the domain $\Omega$, whilst the objective is focused on a given subregion $\sigma$, where $\sigma \subset \Omega$. This approach gave rise to a new research field.

The usual concepts were reconsidered from another point of view in the case of linear systems. In the case of controllability, if we consider the spatial evolution of a distributed parameter system in a domain $\Omega \subset \mathbb{R}^{n}$ and in its subdomain $\sigma \subset \Omega$, given a regional desired state in $\sigma$, the regional controllability (or $\sigma$-controllability) consi- sts in steering the system from its initial state to a desired state, while the objective is focused on $\sigma$. This notion is useful since it has been shown that there exist systems which are not controllable but which are regionally controllable. Moreover, the transfer of a system from its initial state to a desired final state requires a lower cost (Afifi et al., 2008). In addition, as the regional analysis is developed, its ability to deal with real complex systems is extended and becomes deeper.

In 1994, applications to environmental systems led to study the case where the properties of the region under consideration may evolve in time. It may increase or decrease in a sense to be made precise. In some biogeographic processes, regions where a given property is satisfied such as a certain biomass density (in vegetation dynamics), fire expansion, deforestation, immunology, etc. can be identified. The spreadability concept, which describes the expansion process, was then born. Given a property $\mathcal{P}$, we say that a system $(S)$ is $\mathcal{P}$ spreadable if the subregions $\left(\omega_{t}\right)_{t \in I}$ of $\Omega$ satisfying the property $\mathcal{P}$ are increasing in time (El Jai and Kassara, 1994). The usefulness of the spreadability concept justifies the increasing interest in it, and this turns out to necessitate additional mathematical investigations. A concept which naturally follows from the spreadability notion is that of spray control. That is a control which makes the system spreadable (Bernoussi and El Jai, 2000; Bernoussi et al., 2001; El Jai and Kassara, 1994). It was 
made meaningful and then explored by introducing the idea of spread.

As it is almost impossible to stop the spreadability of some processes such as epidemic or desert expansion, one could be interested in the remediability of a given subdomain. Specifically, given an expansion phenomenon $\mathcal{P}$, such as an epidemic or a fire, is a given zone $\sigma$ vulnerable? That is, vulnerable in the sense that it will be affected by the expansion of the property $\mathcal{P}$ (Bernoussi, 2007).

When a system is subjected to disturbances, then one of the objectives becomes to find controls which compensate the effect of the disturbance. In the case of a distributed parameter system, the disturbance can be located in a given subregion $\omega$ of the space domain $\Omega$ while the control is active in another subregion $D$ of $\Omega$. The problem of finding a control in $D$ which would compensate the effects of the disturbance located in $\omega$ is the space compensation problem, i.e., regional remediability. The notion of remediability was introduced in the case of linear systems for both finite and infinite time horizons. Weak and exact regional asymptotic remediability and regionally asymptotic efficient actuators were introduced as well. Various characterizations and an application were investigated and reported in (Afifi et al., 2008; Afifi et al., 2002).

Several other works in this area were devoted to the study of controllability (Doubova et al., 2006) and disturbance compensation (Christofides, 2001) in nonlinear distributed systems. Thus, under some hypothesis on the system dynamics and the nonlinearity term, the problem of control is studied using global Carleman estimates, parabolic regularity and the fixed point method when the nonlinear term involves the state and the gradient (Doubova et al., 2006). Moreover, an approach using direct space discretization (Christofides, 1998; Christofides and Daoutidis, 1998; Christofides, 2001) is given for the second problem. In this paper, the problem considered is different in the sense that it consists in an extension of the regionally remediability concept to a class of nonlinear distributed parameter systems in the case where the system is subject to an initial expanding disturbance, i.e., a disturbance that happens in $\omega$ at the initial time only, as shown in the left part of Fig. 1(a) and will spread over the domain and may affect a part of the domain (the right part of Fig. 1(b)) or the whole domain.

Consequently, given a subregion $\sigma$, the disturbance effect can reach $\sigma$, see Fig. 1(b). In this paper we consider an extension of remediability which neutralizes the effect of the disturbance on $\sigma$, i.e., the disturbance effect changes from Fig. 1 (b) to Fig. 2

The paper is organized as follows: The next section is devoted to the problem statement of the regional remediability of a class of nonlinear systems. We recall and extend the remediability definition to the case of nonlinear systems. In Section 3, we consider some mathematical results which lead to a new problem statement that is more

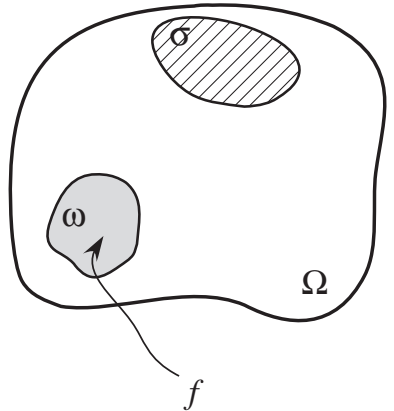

(a)

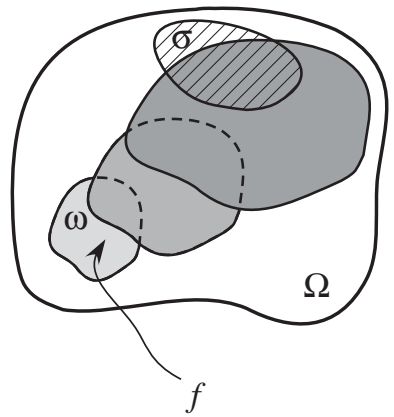

(b)

Fig. 1. Initial disturbance in $\omega$ (a) and disturbance expansion in $\Omega$ (b).

useful for implementation. Can we find a control which makes it possible to compensate such a disturbance at a certain time $T$ ? This is considered in Section 4, where we show how the problem may be solved using pseudo inverse techniques and the fixed point theorem. In the last section, we give an algorithmic scheme and illustrate the developed approach by a one-dimensional example.

Let $\Omega$ be an open bounded domain of $\mathbb{R}^{n}$ and $I=$ ]0,T a time interval. Consider a distributed system whose state $\widetilde{z}(x, t)$ satisfies a nonlinear equation in $\Omega \times I$, and the initial state $\widetilde{z}(x, 0)=z_{0}(x)$ is subject to a space di-

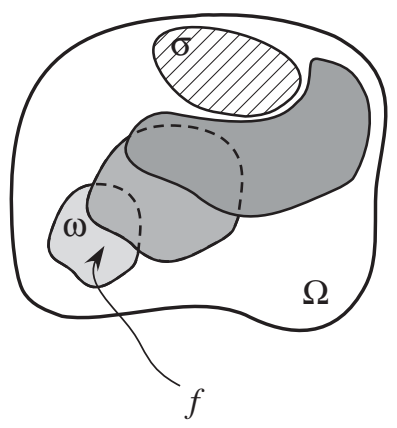

Fig. 2. Remediability of the region $\sigma$. 
sturbance $f$ defined on $\Omega$,

$$
(\widetilde{S})\left\{\begin{array}{l}
\dot{\widetilde{z}}(t)=F \widetilde{z}(t)+B u(t), \quad 0<t<T, \\
\widetilde{z}(0)=\left(z_{0}+f\right) \in \mathcal{D}(F),
\end{array}\right.
$$

where $\widetilde{z} \in L^{p}(0, T ; Z)$ for $\left.p \in\right] 1,+\infty\left[, u \in L^{2}(0, T ; \mathcal{U})\right.$, $f \in \mathcal{F}, B \in \mathcal{L}(\mathcal{U} ; Z)$, and $F: X=\mathcal{D}(F) \rightarrow Z$ is a nonlinear operator with the domain $X$ being a Banach space dense in $Z$ and satisfying the assumptions

1. $F(0)=0$,

2. $F \in C^{1}$ in $f$ for $f \in \mathcal{D}(F)$.

The state space $Z$, the control space $\mathcal{U}$, and the disturbance space $\mathcal{F}$ are assumed to be separable Hilbert spaces, and $\mathcal{F} \subset \mathcal{D}(F)$.

Let $\sigma$ be a fixed subregion in $\Omega$. Denote by $\chi_{\sigma}$ the restriction to $\sigma$ and consider the set of restriction states to $\sigma$ given by

$$
Z_{\sigma}=\left\{\chi_{\sigma} z(t)=\left.z(t)\right|_{\sigma}: z(t) \in Z\right\}, \quad \forall t \in I .
$$

It is clear that $Z_{\sigma}$ is a subspace of the state space $Z$. Usually, $Z=L^{2}(\Omega)$, and then $Z_{\sigma}=L^{2}(\sigma)$. The adjoint operator of $\chi_{\sigma}$ is defined by

$$
\chi_{\sigma}^{*}: Z_{\sigma} \rightarrow Z
$$

such that

$$
\chi_{\sigma}^{*} z= \begin{cases}z & \text { in } \sigma \\ 0 & \text { elsewhere. }\end{cases}
$$

The system (1) is augmented by the output function

$$
(\widetilde{E}) \quad \widetilde{y}^{\sigma}(t)=C \chi_{\sigma}^{*} \chi_{\sigma} \widetilde{z}(t),
$$

where $C \in \mathcal{L}(Z ; Y)$ and $Y$ is a separable Hilbert space (the observation space).

We assume that the disturbance $f$ is spreadable from $\omega$ and may spread to the whole domain $\Omega$. Thus the spreadability effect can reach the subregion $\sigma$, which is then said to be $f$-vulnerable.

Remark 1. In (1), the disturbance function $f$ has a space support which can be, in practical applications, a part $\omega$ of the domain $\Omega(\omega \subseteq \Omega)$. It can also be written in the form $f(x)=\chi_{\omega} g$ where $g$ is defined in $\Omega$, so the disturbance $f$ can be considered as defined in all $\Omega$, assuming that it vanishes in $\Omega \backslash \omega$. This initial state in its form (1) is more convenient for the spreadability description. In both cases the whole domain $\Omega$ will be affected by the effect of the disturbance $f$.

\section{Problem statement}

In this paper we explore the possibility of finding a control $u$ yielding a regional compensation of the space disturbance $f$ at some time $T$, in the sense that the system output becomes, at time $T$, equal to the output of a nondisturbed system. Under the effect of the space disturbance $f$, which affects the initial state, the disturbed system $(f \neq 0$ and $u=0$ ) becomes $\mathcal{P}$-spreadable where $\mathcal{P}$ is a property generated by the disturbance $f$. Consequently, the fixed subregion $\sigma$ can be vulnerable (i.e., likely to be reached by the property $\mathcal{P}$ ) (Bernoussi and Amharref, 2003). How can we determine a control allowing us to neutralize the spreadability of the disturbed system on $\sigma$ at the final time $T$ ? This is the principle of the regional remediability problem.

We assume that the system $(\widetilde{S})$ has a unique solution denoted by $\widetilde{z}_{f, u}$ and we adopt the following notation:

$$
\begin{cases}\widetilde{z}_{f, u} & \text { solution of }(\widetilde{S}), \\ \widetilde{z}_{f, 0} & \text { solution of }(\widetilde{S}) \text { when } u=0 \text { and } f \neq 0, \\ \widetilde{z}_{0,0} & \text { solution of }(\widetilde{S}) \text { when } u=0 \text { and } f=0, \\ \widetilde{y}_{f, u}^{\sigma} & \text { output }(\widetilde{E}), \\ \widetilde{y}_{f, 0}^{\sigma} & \text { output }(\widetilde{E}) \text { when } u=0 \text { and } f \neq 0, \\ \widetilde{y}_{0,0}^{\sigma} & \text { output }(\widetilde{E}) \text { when } u=0 \text { and } f=0 .\end{cases}
$$

Thus $\widetilde{z}_{f, 0}$ is the solution to the disturbed and autonomous system, i.e., $f \neq 0$ and $u=0$, while $\widetilde{y}_{f, 0}^{\sigma}$ is the associated output. Given the property $\mathcal{P}$, consider the sets

$$
w_{f, 0, t}=\left\{x \in \Omega: \mathcal{P} \widetilde{z}_{f, 0}(x, t)\right\},
$$

where $w_{f, 0, t}$ denotes the set of points $x \in \Omega$ where the state of the disturbed system $\widetilde{z}_{f, 0}$ satisfied the property $\mathcal{P}$ at the time $t$. Let $\mu(E)=\int_{E} \mathrm{~d} x$ signify the Lebesgue measure. We recall the following definition (Qaraai et al., 2008):

\section{Definition 1.}

1. We say that the disturbance $f$ is spreadable (respectively, $\mathcal{A}$-spreadable $)$ if the family $\left(w_{f, 0, t}\right)_{t \in I}$ is increasing in the inclusion sense,

$$
w_{f, 0, t} \subset w_{f, 0, s}, \quad \forall t, s \in I, \quad t \leq s,
$$

(resp. in the sense of measure,

$$
\begin{array}{r}
\mu\left(w_{f, 0, t} / w_{f, 0, s}\right) \leq \mu\left(w_{f, 0, s} / w_{f, 0, t}\right), \\
\forall t, s \in I, \quad t \leq s) .
\end{array}
$$

2. The subregion $\sigma$ is said to be $f$-vulnerable if there exists $t \in[0, T]$ such that

$$
\stackrel{\circ}{\sigma} \cap w_{f, 0, t} \neq \emptyset
$$


Note that the vulnerability definition is based on the interior $\stackrel{\circ}{\sigma}$ instead of $\sigma$ because the boundary $\Gamma_{\sigma}$ can be a natural barrier which can stop the spreadability of $f$.

Thus for the initial state $z_{0} \in X$ and the disturbance $f \in \mathcal{F}$, using the output $(\widetilde{E})$ and Definition 1 we consider the following definitions:

Definition 2. If the subregion $\sigma$ is $f$-vulnerable, then: 1 . The system $(\widetilde{S})$ is said to be exactly $f$ - $\sigma$-remediable if

$\exists u \in L^{2}(0, T ; \mathcal{U})$ such that $\widetilde{y}_{f, u}^{\sigma}(T)=\widetilde{y}_{0,0}^{\sigma}(T)$.

2. The system $(\widetilde{S})$ is said to be weakly $f$ - $\sigma$-remediable if $\exists u \in L^{2}(0, T ; \mathcal{U})$ such that $\left\|\widetilde{y}_{f, u}^{\sigma}(T)-\widetilde{y}_{0,0}^{\sigma}(T)\right\|_{Y} \leq \varepsilon$.

In the above, Case 1 concerns the case where the control $u$ compensates in $\sigma$ the effect of the disturbance $f$ at time $T$. In Case 2 the control allows the weak neutralization of the effect of $f$ in the subregion with a tolerance $\operatorname{margin} \varepsilon$.

From the above definition we can state the remediability problem as follows:

$$
(\widetilde{P})\left\{\begin{array}{l}
\text { Find } u \in L^{2}(0, T ; \mathcal{U}) \text { such that } \\
\widetilde{y}_{f, u}^{\sigma}(T)=\widetilde{y}_{0,0}^{\sigma}(T) .
\end{array}\right.
$$

The solution of $(\widetilde{P})$ is equivalent to finding a control $u$ which makes the system $(\widetilde{S})$ (and then the output $(\widetilde{E})$ ) normal in $\sigma$ at time $T$. In other words, this is as if we had $\widetilde{y}_{0,0}^{\sigma}=C \chi_{\sigma}^{*} \chi_{\sigma} \widetilde{z}_{0,0}$, where $\widetilde{z}_{0,0}$ is the solution of the following uncontrolled nondisturbed system:

$$
\left\{\begin{array}{l}
\dot{\widetilde{z}}(t)=F \widetilde{z}(t), \quad 0<t<T, \\
\widetilde{z}(0)=z_{0} .
\end{array}\right.
$$

Problem $(\widetilde{P})$ is not easy to solve. Indeed, on the one hand the solution $\widetilde{z}_{f, u}$ of the system $(\widetilde{S})$ is not known. On the other hand, this solution does not take into account explicitly the control $u$ and the disturbance $f$, for applying the remediability results developed in (Afifi et al., 2002). To overcome these difficulties, a linearization of the system $(\widetilde{S})$ and the output $(\widetilde{E})$ will be considered in the neighborhood of the disturbance $f$. Therefore we use a semigroup approach (Curtain and Pritchard, 1978; Dautray and Lions, 1984; Pazy, 1983), pseudo inverse techniques (El Jai et al., 1986) and the fixed-point theorem for exploring the solution of Problem $(\widetilde{P})$.

Firstly, considering the variable change $z(t)=$ $\widetilde{z}(t)-f$, the system (1) becomes

$$
\left\{\begin{array}{l}
\dot{z}(t)=F(z(t)+f)+B u(t), \quad 0<t<T, \\
z(0)=z_{0} .
\end{array}\right.
$$

Using Assumption 2 on $F$ and linearizing around $f$, we obtain

$$
F(z(t)+f)=F f+F^{\prime}(f) z(t)+N_{f} z(t),
$$

which leads to the linearized system

$$
(S)\left\{\begin{aligned}
\dot{z}(t) & =A_{f} z(t)+N_{f} z(t)+F f+B u(t), \\
& 0<t<T, \\
z(0) & =z_{0},
\end{aligned}\right.
$$

where $A_{f}=F^{\prime}(f) \in \mathcal{L}(Z)$ is the linear part of the dynamic $F$ with a domain $\mathcal{D}\left(A_{f}\right)$ densely included in the state space $Z$, and $N_{f}: z(t) \in X \mapsto N_{f} z(t) \in Z$ denotes the nonlinear term defined only in a Banach space $X$ with an image in $Z$ such that $X \subset Z$. Notice that if $N_{f}=0$, then $F=A_{f}$ and $X=Z$.

In the vicinity of $f$ the output function $(\widetilde{E})$ becomes

$$
\widetilde{y}^{\sigma}(t)=C \chi_{\sigma}^{*} \chi_{\sigma} \widetilde{z}(t)=C \chi_{\sigma}^{*} \chi_{\sigma}(z(t)+f) .
$$

Let $y^{\sigma}(t)=\widetilde{y}^{\sigma}(t)-C \chi_{\sigma}^{*} \chi_{\sigma} f$. Then, since $C \chi_{\sigma}^{*} \chi_{\sigma}$ is linear, the output becomes

$$
\text { (E) } y^{\sigma}(t)=C \chi_{\sigma}^{*} \chi_{\sigma} z(t) .
$$

Finally, in the neighborhood of the disturbance $f$, we obtain the following new formulation of the remediability problem:

$$
(P)\left\{\begin{array}{l}
\text { Find } u \in L^{2}(0, T ; \mathcal{U}) \text { such that } \\
y_{f, u}^{\sigma}(T)=y_{0,0}^{\sigma}(T)-C \chi_{\sigma}^{*} \chi_{\sigma} f .
\end{array}\right.
$$

Remark 2. At this juncture, the following conclusions can be drawn:

1. Problem $(\widetilde{P})$ is equivalent to $(P)$ in the neighborhood of the disturbance $f$.

2. In the case when the system (1) evolves on a time interval $\left[0, T^{*}\right]$, where $T^{*}>T$, at time $T$ the disturbance is neutralized, and therefore the system will evolve normally on $\left[T, T^{*}\right]$ since the effect of the disturbance has been removed.

3. Observe that in the case where $\sigma=\Omega$, Problem $(P)$ is a global remediability problem. It consists in compensating the disturbance $f$ in the whole domain $\Omega$ because the domain is necessarily $f$-vulnerable.

4. If the subregion $\sigma$ is not $f$-vulnerable, then we do not have to remedy $f$.

The solution of Problem $(P)$ needs an explicit formulation in $f, u$ and $z_{f, u}$. This will be considered in the next section. 


\section{Regional remediability formulation}

In all what follows, we denote by $\|\cdot\|_{p}=\|\cdot\|_{L^{p}(0, T ; X)}$, for $p \in] 1,+\infty\left[\right.$. Suppose that the linear operator $A_{f}$ is closed with a domain densely included in $Z$ and generates a strongly continuous semigroup $\left(S_{f}(t)\right)_{t \geq 0}$ which satisfies the assumption

$$
\text { (A1) } \begin{aligned}
& \exists g_{1} \in L^{q}(0, T) \text { with } \frac{1}{q}+\frac{1}{p}=1: \\
&\left\|S_{f}(t)\right\|_{\mathcal{L}(Z)} \leq g_{1}(t), \quad \forall t \in[0, T] .
\end{aligned}
$$

Moreover, let the nonlinear operator $N_{f}: X \rightarrow Z$ be well defined and satisfy the following assumption:

(A2)

$$
\left\{\begin{array}{l}
\text { 1. } N_{f}(0)=0 \\
\text { 2. }\left\|N_{f} z_{1}(t)-N_{f} z_{2}(t)\right\|_{Z} \\
\quad \leq K\left(\left\|z_{1}\right\|_{p} ;\left\|z_{2}\right\|_{p}\right)\left\|z_{1}(t)-z_{2}(t)\right\|_{X} \\
\quad \forall z_{1}, z_{2} \in L^{p}(0, T ; X) ; K: \\
\mathbb{R}_{+}^{2} \longrightarrow \mathbb{R}_{+}: \lim _{\left(\theta_{1} ; \theta_{2}\right) \rightarrow(0,0)} K\left(\theta_{1} ; \theta_{2}\right)=0
\end{array}\right.
$$

For a given $a \in] 0,1[$ we set

$$
\begin{aligned}
k & =\sup _{\theta<a} K(\theta ; 0), \\
K & =\sup _{\left\|\left(\theta_{1} ; \theta_{2}\right)\right\|_{\mathbb{R}_{+}^{2}<a}} K\left(\theta_{1} ; \theta_{2}\right), \\
K^{*} & =\max (k, K) .
\end{aligned}
$$

Under these assumptions, and with some others that will be indicated below, we show that the unique solution to the system $(S)$ can be written in the form

$$
\begin{aligned}
z_{f, u}(t)= & S_{f}(t) z_{0}+\int_{0}^{t} S_{f}(t-s) N_{f} z_{f, u}(s) \mathrm{d} s \\
& +\int_{0}^{t} S_{f}(t-s) F f \mathrm{~d} s \\
& +\int_{0}^{t} S_{f}(t-s) B u(s) \mathrm{d} s
\end{aligned}
$$

or, equivalently,

$$
z_{f, u}(t)=S_{f}(t) z_{0}+\widehat{H}_{f, t} z_{f, u}+\widetilde{H}_{f, t} f+H_{f, t} u,
$$

where the three operators have images in $Z$ and act respectively on the state, disturbance and control variables. They are expressed by

$$
\begin{aligned}
\widehat{H}_{f, t}:\left\{\begin{aligned}
L^{p}(0, T ; X) & \rightarrow Z \\
z & \mapsto \int_{0}^{t} S_{f}(t-s) N_{f} z(s) \mathrm{d} s
\end{aligned}\right. \\
\widetilde{H}_{f, t}:\left\{\begin{aligned}
\mathcal{F} & \rightarrow Z \\
f & \mapsto \int_{0}^{t} S_{f}(t-s) F f \mathrm{~d} s
\end{aligned}\right.
\end{aligned}
$$

$$
H_{f, t}:\left\{\begin{aligned}
L^{2}(0, T ; \mathcal{U}) & \rightarrow Z \\
u & \mapsto \int_{0}^{t} S_{f}(t-s) B u(s) \mathrm{d} s .
\end{aligned}\right.
$$

Moreover, we consider the mapping $\psi(\cdot, f, u): z \in$ $L^{p}(0, T ; X) \longmapsto \psi(z, f, u) \in L^{p}(0, T ; X)$ defined by

$$
\psi(z, f, u):\left\{\begin{aligned}
{[0, T] } & \rightarrow X, \\
t & \mapsto \psi(z, f, u)(t)=S_{f}(t) z_{0} \\
& +\widehat{H}_{f, t} z+\widetilde{H}_{f, t} f+H_{f, t} u .
\end{aligned}\right.
$$

Then, to prove that 10 is well defined amounts to proving that under some hypothesis there exists $d>0$ such that the mapping $\psi(\cdot, f, u)$ has one unique fixed point $z_{f, u}$ in a ball $B(0, d)$ of $L^{p}(0, T ; X)$.

For this reason we assume the following:

$$
\left\{\begin{array}{c}
\exists \alpha>0:\left\|S_{f}(t) z_{0}\right\|_{X} \leq \alpha\left\|z_{0}\right\|_{X}, \\
\forall t \in[0, T], \\
\exists \beta>0:\left\|H_{f, t} u\right\|_{X} \leq \beta\|u\|_{L^{2}(0, T ; \mathcal{U})}, \\
\forall t \in[0, T], \\
\exists \gamma>0:\left\|\widetilde{H}_{f, t} f\right\|_{X} \leq \gamma\|f\|_{\mathcal{F}}, \quad \forall t \in[0, T],
\end{array}\right.
$$

(A4) $T<\frac{1}{\left(K^{*}\left\|g_{1}\right\|_{q}\right)^{p}}$,

(A5) $\exists \lambda \in] 0,1[$ :

$$
\left.\alpha\left\|z_{0}\right\|_{X}+\gamma\|f\|_{\mathcal{F}} \in\right] 0, \frac{\lambda}{T^{\frac{1}{p}}}\left(1-T^{\frac{1}{p}} k\left\|g_{1}\right\|_{q}\right)[.
$$

Before stating the principal result of this section, let us interpret the various assumptions considered above.

\section{Remark 3.}

1. The assumption (A1) is true for every strongly continuous semigroup $\left(S_{f}(t)\right)_{t \in I}$ such that $\left\|S_{f}(t)\right\|_{\mathcal{L}(Z)} \leq M e^{w t}$ for some given constants $M$ and $w$ (Curtain and Pritchard, 1978; Pazy, 1983).

2. For the assumption (A2) the nonlinear operator $N_{f}$ is supposed to be globally Lipschitzian on the Banach space $X$.

3. The positive constants $\alpha, \beta$ and $\gamma$ involved in the assumption (A3) are given respectively by

$$
\begin{aligned}
& \alpha=\max _{t \in[0, T]} g_{1}(t), \\
& \beta=\left\|g_{1}\right\|_{q}\|B\|, \\
& \gamma=T^{\frac{1}{p}}\left\|g_{1}\right\|_{q}\|F\| .
\end{aligned}
$$


4. The hypotheses (A4) and (A5) are the conditions on the final time $T$, the initial state $z_{0}$ and the disturbance $f$ for which the state $z_{f, u}$, given in (10), is well defined for some control $u$.

Theorem 1. If (A1)-(A5) are satisfied, then we have the following:

(a) There exist $d>0$ and $m=m(d)>0$ such that for all $u \in B(0, m) \subset L^{2}(0, T ; \mathcal{U})$ the mapping $\psi(\cdot, f, u)$ has one unique fixed point $z_{f, u} \in$ $B(0, d) \subset L^{p}(0, T ; X)$.

(b) The mapping $h_{1}: B(0, m) \rightarrow B(0, d)$ defined by $h_{1}(u)=z_{f, u}$ is Lipschitzian.

Proof. (a) The second condition of (A2), i.e.,

$$
\lim _{\left(\theta_{1} ; \theta_{2}\right) \rightarrow(0,0)} K\left(\theta_{1} ; \theta_{2}\right)=0
$$

implies

$$
\forall \varepsilon>0, \exists d \in] 0,1\left[\sup _{\left\|\left(\theta_{1} ; \theta_{2}\right)\right\|<d} K\left(\theta_{1} ; \theta_{2}\right)<\varepsilon,\right.
$$

where $\|(\cdot ; \cdot)\|$ is a norm on $\mathbb{R}_{+}^{2}$. In particular, for

$$
\varepsilon=\frac{-1}{T^{\frac{1}{p}}\left\|g_{1}\right\|_{q}}
$$

where $g_{1}$ is the function involved in (A1), and using the constant $K$ given by (9), we have

$$
\left.c_{1}=T^{\frac{1}{p}}\left\|g_{1}\right\|_{q} K \in\right] 0,1[.
$$

Remark 4. Note that the choice of the positive constant $\varepsilon$ is not arbitrary, but it is a direct consequence of (A4), which implies $\left.c_{1} \in\right] 0,1[$.

First, for $f \in \mathcal{F}$ and $u \in L^{2}(0, T ; \mathcal{U})$, let us prove that $\psi(B(0, d), f, u) \subset B(0, d)$. Indeed, for $z \in B(0, d)$ and $\forall t \in[0, T]$ we have

$$
\begin{aligned}
&\|\psi(z, f, u)(t)\|_{X} \\
&=\|\psi(z, f, u)(t)-\psi(0, f, u)(t)+\psi(0, f, u)(t)\|_{X} \\
&=\left\|\widehat{H}_{f, t} z-\widehat{H}_{f, t}(0)+\psi(0, f, u)(t)\right\|_{X} \\
& \leq\left\|\widehat{H}_{f, t} z-\widehat{H}_{f, t}(0)\right\|_{X}+\|\psi(0, f, u)(t)\|_{X} \\
& \leq \int_{0}^{t}\left\|S_{f}(t-s)\left[N_{f} z(s)-N_{f}(0)(s)\right]\right\|_{X} \mathrm{~d} s \\
& \quad+\|\psi(0, f, u)(t)\|_{X} \\
& \leq \int_{0}^{t} g_{1}(t-s)\left\|\left[N_{f} z(s)-N_{f}(0)(s)\right]\right\|_{X} \mathrm{~d} s \\
&+\|\psi(0, f, u)(t)\|_{X},
\end{aligned}
$$

with $g_{1}$ given in (A1). If we consider the function $g$ defined by

$$
g:\left\{\begin{aligned}
{[0, t] } & \rightarrow \mathbb{R}_{+}^{*}, \\
s & \mapsto g(s)=g_{1}(t-s),
\end{aligned}\right.
$$

then it is clear that $g \in L^{q}(0, T)$ and $\|g\|_{q}=\left\|g_{1}\right\|_{q}$. Consequently, using the Hölder inequality and Assumptions (A2) and (A3), we have

$$
\begin{aligned}
\|\psi(z, f, u)(t)\|_{X} \leq & \alpha\left\|z_{0}\right\|_{X}+\beta\|u\|_{L^{2}(0, T ; \mathcal{U})} \\
& +\gamma\|f\|_{\mathcal{F}}+\left\|g_{1}\right\|_{q} K\left(\|z\|_{p} ; 0\right)\|z\|_{p} .
\end{aligned}
$$

Then

$$
\begin{aligned}
\int_{0}^{T}\|\psi(z, f, u)(t)\|_{X}^{p} \mathrm{~d} t \leq & T\left(\alpha\left\|z_{0}\right\|_{X}+\beta\|u\|_{L^{2}(0, T ; \mathcal{U})}\right. \\
& +\gamma\|f\|_{\mathcal{F}}+\left\|g_{1}\right\|_{q} \\
& \left.\times K\left(\|z\|_{p} ; 0\right)\|z\|_{p}\right)^{p}
\end{aligned}
$$

which gives, using the norm $\|\cdot\|_{p}$,

$$
\begin{aligned}
\|\psi(z, f, u)\|_{p} \leq & T^{\frac{1}{p}}\left(\alpha\left\|z_{0}\right\|_{X}+\beta\|u\|_{L^{2}(0, T ; \mathcal{U})}\right. \\
& \left.+\gamma\|f\|_{\mathcal{F}}+\left\|g_{1}\right\|_{q} K\left(\|z\|_{p} ; 0\right)\|z\|_{p}\right) .
\end{aligned}
$$

Thus the condition

$$
z \in B(0, d) \Rightarrow \psi(z, f, u) \in B(0, d)
$$

is satisfied (using the constant $k$ given by (9)) when

$T^{\frac{1}{p}}\left(\alpha\left\|z_{0}\right\|_{X}+\beta\|u\|_{L^{2}(0, T ; \mathcal{U})}+\gamma\|f\|_{\mathcal{F}}+k\left\|g_{1}\right\|_{q} d\right) \leq d$.

That is to say,

$\|u\|_{L^{2}(0, T ; \mathcal{U})}$

$$
\leq \frac{1}{\beta}\left[\frac{d\left(1-T^{\frac{1}{p}} k\left\|g_{1}\right\|_{q}\right)}{T^{\frac{1}{p}}}-\alpha\left\|z_{0}\right\|_{X}-\gamma\|f\|_{\mathcal{F}}\right] .
$$

Finally, we obtain

$$
\|u\|_{L^{2}(0, T ; \mathcal{U})} \leq m
$$

with

$$
m=\frac{1}{\beta}\left[\frac{d\left(1-T^{\frac{1}{p}} k\left\|g_{1}\right\|_{q}\right)}{T^{\frac{1}{p}}}-\alpha\left\|z_{0}\right\|_{X}-\gamma\|f\|_{\mathcal{F}}\right] .
$$

The assumption $(A 4)$ implies $1-T^{\frac{1}{p}} k\left\|g_{1}\right\|_{q}>0$. Then, using $(A 5)$ for $\lambda=d \in] 0,1[$, we have $m>0$. Thus for $u \in B(0, m)$ we obtain $\psi(B(0, d), f, u) \subset B(0, d)$.

On the other hand, for $f \in \mathcal{F}$ and $u \in L^{2}(0, T ; \mathcal{U})$ we prove that $\psi(\cdot, f, u)$ is a contraction in $B(0, d)$. Let $z_{1}, z_{2} \in B(0, d)$. Then for every $t \in I$ we have

$$
\begin{aligned}
& \left\|\psi\left(z_{1}, f, u\right)(t)-\psi\left(z_{2}, f, u\right)(t)\right\|_{X} \\
& \quad=\left\|\widehat{H}_{f, t} z_{1}-\widehat{H}_{f, t} z_{2}\right\|_{X} \\
& \quad=\left\|\int_{0}^{t} S_{f}(t-s)\left[N_{f} z_{1}(s)-N_{f} z_{2}(s)\right] \mathrm{d} s\right\|_{X},
\end{aligned}
$$


where $\widehat{H}_{f, t}$ and $\psi(z, f, u)$ are respectively given by (12) and 15 . obtain

Using (A1) and (A2) and the Hölder inequality, we

$$
\begin{aligned}
& \left\|\psi\left(z_{1}, f, u\right)(t)-\psi\left(z_{2}, f, u\right)(t)\right\|_{X} \\
& \quad \leq\left\|g_{1}\right\|_{q} K\left(\left\|z_{1}\right\|_{p} ;\left\|z_{2}\right\|_{p}\right)\left\|z_{1}-z_{2}\right\|_{p} .
\end{aligned}
$$

That is to say,

$$
\begin{aligned}
& \int_{0}^{T}\left\|\psi\left(z_{1}, f, u\right)(t)-\psi\left(z_{2}, f, u\right)(t)\right\|_{X}^{p} \mathrm{~d} t \\
& \quad \leq T\left(\left\|g_{1}\right\|_{q} K\left(\left\|z_{1}\right\|_{p} ;\left\|z_{2}\right\|_{p}\right)\left\|z_{1}-z_{2}\right\|_{p}\right)^{p} .
\end{aligned}
$$

Then, using the norm $\|\cdot\|_{p}$ and the constant $c_{1}$ given in (16), we have

$$
\left\|\psi\left(z_{1}, f, u\right)-\psi\left(z_{2}, f, u\right)\right\|_{p} \leq c_{1}\left\|z_{1}-z_{2}\right\|_{p},
$$

which means that $\psi(\cdot, f, u)$ is a contraction on $B(0, d)$ as $\left.c_{1} \in\right] 0,1[$

Thus, if $u \in B(0, m)$ of $L^{2}(0, T ; \mathcal{U})$, the mapping $\psi(\cdot, f, u)$ has a unique fixed point $z_{f, u}$ in a ball $B(0, d)$ of $L^{p}(0, T ; X)$, which is the solution to the system $(S)$.

(b) Let $u, v \in B(0, m)$. Then, using $\psi(\cdot, f, u), h_{1}$ and (A3), we have

$$
\begin{aligned}
\| h_{1}(u)- & h_{1}(v) \|_{p} \\
= & \left\|\psi\left(h_{1}(u), f, u\right)-\psi\left(h_{1}(v), f, v\right)\right\|_{p} \\
\leq & \left\|\psi\left(h_{1}(u), f, u\right)-\psi\left(h_{1}(v), f, u\right)\right\|_{p} \\
& \quad+\left\|\psi\left(h_{1}(v), f, u\right)-\psi\left(h_{1}(v), f, v\right)\right\|_{p} \\
\leq & c_{1}\left\|h_{1}(u)-h_{1}(v)\right\|_{p}+T^{\frac{1}{p}} \beta\|u-v\|_{L^{2}(0, T ; \mathcal{U})} \\
\leq & \frac{T^{\frac{1}{p}} \beta}{1-c_{1}}\|u-v\|_{L^{2}(0, T ; \mathcal{U})},
\end{aligned}
$$

which means that $h_{1}$ is Lipschitzian in $B(0, m)$.

Proposition 1. Under Assumptions (A1)-(A5), the system $(S)$ has a unique weak solution given by (10).

Remark 5. When the linear operator $A_{f}$ is analytic (Henry, 1981), all the results given in Theorem 1 remain valid if we take into account the following: Let $a \in \mathbb{R}$ such that $\operatorname{Re}\left(\operatorname{spec}\left(a I+A_{f}\right)\right)>\delta>0$, where $\operatorname{spec}(M)$ is the spectrum of the linear operator $M$. For $\alpha \in[0,1[$, $Z^{\alpha}=D\left(\left(a I+A_{f}\right)^{\alpha}\right)$ is a Banach space densely included in $Z$ with the graph norm $\|(\cdot)\|_{Z^{\alpha}}=\|(a I+$ $\left.A_{f}\right)^{\alpha}(\cdot) \|_{Z}$. We recall the following result (Henry, 1981):

$$
\exists c>0: \quad\left\|S_{f}(t)\right\|_{\mathcal{L}\left(Z^{\alpha}, Z\right)} \leq c t^{-\alpha} e^{(a-\delta) t}=\bar{g}_{1}(t),
$$$$
\forall t \in I \text {. }
$$

Then for $p \in] 1,+\infty[$ the assumption $(A 1)$ can be replaced by

$$
\overline{(A 1)} \exists q \in] 1,+\infty\left[, \frac{1}{p}+\frac{1}{q}=1, \quad q \alpha<1,\right.
$$

such that $(18)$ is satisfied. Note that the condition $q \alpha<1$ implies $\bar{g}_{1} \in L^{q}(0, T)$. Moreover, the domain $X$ of the nonlinear operator $N_{f}$ is replaced by $Z^{\alpha}$. Then it was shown (Cholewa and Dłotko, 2000) that if $(A 2)$ holds on $Z^{\alpha}$, then it remains true on $Z^{\nu}$ for all $\nu \in[\alpha, 1[$.

In all what follows, we consider only the case where $A_{f}$ is the infinitesimal generator of a strongly continuous semigroup. Then, given the solution to the system $(S)$ around $f$,

$$
z_{f, u}(t)=S_{f}(t) z_{0}+\widehat{H}_{f, t} z_{f, u}+\widetilde{H}_{f, t} f+H_{f, t} u,
$$

we can rewrite the output function $(E)$ as follows:

$$
\begin{aligned}
y_{f, u}^{\sigma}(t)= & C \chi_{\sigma}^{*} \chi_{\sigma} S_{f}(t) z_{0}+C \chi_{\sigma}^{*} \chi_{\sigma} \widehat{H}_{f, t} z_{f, u} \\
& +C \chi_{\sigma}^{*} \chi_{\sigma} \widetilde{H}_{f, t} f+C \chi_{\sigma}^{*} \chi_{\sigma} H_{f, t} u .
\end{aligned}
$$

In the case where $f=0$ and $u=0$, we have

$$
z_{0,0}(t)=S_{0}(t) z_{0}+\widehat{H}_{0, t} z_{0,0} .
$$

The associated output function is

$y_{0,0}^{\sigma}(t)=C \chi_{\sigma}^{*} \chi_{\sigma} S_{0}(t) z_{0}+C \chi_{\sigma}^{*} \chi_{\sigma} \widehat{H}_{0, t} z_{0,0}$.

Consequently, Problem $(P)$ can be stated in the following form:

$$
(P)\left\{\begin{array}{l}
\text { Find } u \in L^{2}(0, T ; \mathcal{U}) \text { such that } \\
C \chi_{\sigma}^{*} \chi_{\sigma} \widehat{H}_{f, T} z_{f, u}+C \chi_{\sigma}^{*} \chi_{\sigma} \widetilde{H}_{f, T} f \\
+C \chi_{\sigma}^{*} \chi_{\sigma} f+C \chi_{\sigma}^{*} \chi_{\sigma} H_{f, T} u \\
+C \chi_{\sigma}^{*} \chi_{\sigma} S_{f}(T) z_{0}-C \chi_{\sigma}^{*} \chi_{\sigma} S_{0}(T) z_{0} \\
-C \chi_{\sigma}^{*} \chi_{\sigma} \widehat{H}_{0, T} z_{0,0}=0 .
\end{array}\right.
$$

\section{Remark 6.}

1. If $f=0$, then the control $u=0$ is the solution to $(P)$, i.e., when the system is not disturbed, no remediability is needed.

2. If $N=0$, i.e., $\widehat{H}_{f, T}=0$, we retrieve the formulation given in the linear case in (Afifi et al., 2002).

3. The established results have a local character. This is a consequence of the linearization of $F$ in the neighborhood of $f$.

\section{Problem solution}

The solution of Problem $(P)$ consists in finding a control $u$ which compensates the effect of the disturbance $f$. This can be solved, under some assumptions, using pseudoinverse techniques (El Jai et al., 1986) and the fixed-point theorem. This leads to a sequence of controls which converges to the solution of $(P)$. 
A control $u$ is a solution to $(P)$ if it satisfies

$$
\begin{aligned}
& C \chi_{\sigma}^{*} \chi_{\sigma} H_{f, T} u+C \chi_{\sigma}^{*} \chi_{\sigma} \widehat{H}_{f, T} z_{f, u} \\
& +C \chi_{\sigma}^{*} \chi_{\sigma} \widetilde{H}_{f, T} f+C \chi_{\sigma}^{*} \chi_{\sigma} f+C \chi_{\sigma}^{*} \chi_{\sigma} S_{f}(T) z_{0} \\
& \quad-C \chi_{\sigma}^{*} \chi_{\sigma} S_{0}(T) z_{0}-C \chi_{\sigma}^{*} \chi_{\sigma} \widehat{H}_{0, T} z_{0,0}=0 .
\end{aligned}
$$

Consider the subset $V=\operatorname{Im}\left(C \chi_{\sigma}^{*} \chi_{\sigma} H_{f, T}\right)$ of $Y$ and write

$$
\begin{aligned}
y_{f}^{\sigma}= & C \chi_{\sigma}^{*} \chi_{\sigma} \widehat{H}_{0, T} z_{0,0}+C \chi_{\sigma}^{*} \chi_{\sigma} S_{0}(T) z_{0} \\
& -C \chi_{\sigma}^{*} \chi_{\sigma} S_{f}(T) z_{0}-C \chi_{\sigma}^{*} \chi_{\sigma} \widetilde{H}_{f, T} f \\
& -C \chi_{\sigma}^{*} \chi_{\sigma} f .
\end{aligned}
$$

Then one seeks a control $u$ such that

$$
C \chi_{\sigma}^{*} \chi_{\sigma} H_{f, T} u+C \chi_{\sigma}^{*} \chi_{\sigma} \widehat{H}_{f, T} z_{f, u}=y_{f}^{\sigma},
$$

where we assume that $y_{f}^{\sigma} \in V$.

Note that when the nonlinear term is zero, i.e., $C \chi_{\sigma}^{*} \chi_{\sigma} \widehat{H}_{f, T} z_{f, u}=0$, the set

$$
\left\{u: C \chi_{\sigma}^{*} \chi_{\sigma} H_{f, T} u=y_{f}^{\sigma}\right\}
$$

is closed, convex and nonempty in $L^{2}(0, T ; \mathcal{U})$. Thus there exists a unique minimum-norm control $u^{*}$ satisfying

$$
C \chi_{\sigma}^{*} \chi_{\sigma} H_{f, T} u^{*}=y_{f}^{\sigma} .
$$

Let $u^{*}=L y_{f}^{\sigma}$. Thus we define a linear operator $L$ mapping $V$ into $L^{2}(0, T ; \mathcal{U})$ which is the pseudo inverse of $C \chi_{\sigma}^{*} \chi_{\sigma} H_{f, T}$,

$$
\begin{aligned}
L= & \left(C \chi_{\sigma}^{*} \chi_{\sigma} H_{f, T}\right)^{\dagger} \\
= & H_{f, T}^{*} \chi_{\sigma}^{*} \chi_{\sigma} C^{*} \\
& \times\left[C \chi_{\sigma}^{*} \chi_{\sigma} H_{f, T} H_{f, T}^{*} \chi_{\sigma}^{*} \chi_{\sigma} C^{*}\right]^{-1},
\end{aligned}
$$

where $H_{f, T}^{*}$ and $C^{*}$ denote respectively the adjoint operators of $H_{f, T}$ and $C$.

The space $V$ can be endowed with the norm

$$
\|(\cdot)\|_{V}=\|L(\cdot)\|_{L^{2}(0, T ; \mathcal{U})} .
$$

The Banach space $\left(V,\|\cdot\|_{V}\right)$ will be considered to study Problem $(P)$.

For $y_{f}^{\sigma} \in V$ and using Theorem 1, we look for a control $u \in B(0, m)$ such that

$$
C \chi_{\sigma}^{*} \chi_{\sigma} H_{f, T} u+C \chi_{\sigma}^{*} \chi_{\sigma} \widehat{H}_{f, T} h_{1}(u)=y_{f}^{\sigma},
$$

where $z_{f, u}=h_{1}(u)$, and the positive constant $m$ is given by 17 .

Equivalently, we have

$$
C \chi_{\sigma}^{*} \chi_{\sigma} H_{f, T} u=y_{f}^{\sigma}-C \chi_{\sigma}^{*} \chi_{\sigma} \widehat{H}_{f, T} h_{1}(u) .
$$

Remark 7. Indeed, we have $C \chi_{\sigma}^{*} \chi_{\sigma} \widehat{H}_{f, T} h_{1}(u) \in V$ when $C \chi_{\sigma}^{*} \chi_{\sigma} S_{f}(t) \in \mathcal{L}(Z, V)$.

Using the above remark, and since $y_{f}^{\sigma} \in V$, we have that $u$ is a solution to $(P)$ if

$$
u=L\left(y_{f}^{\sigma}-C \chi_{\sigma}^{*} \chi_{\sigma} \widehat{H}_{f, T} h_{1}(u)\right)
$$

Now for $y_{f}^{\sigma} \in V$ consider the mapping $\varphi\left(y_{f}^{\sigma}, \cdot\right)$ given by

$$
\varphi\left(y_{f}^{\sigma}, \cdot\right):\left\{\begin{array}{l}
L^{2}(0, T ; \mathcal{U}) \rightarrow L^{2}(0, T ; \mathcal{U}) \\
u \mapsto L\left(y_{f}^{\sigma}-C \chi_{\sigma}^{*} \chi_{\sigma} \widehat{H}_{f, T} h_{1}(u)\right)
\end{array}\right.
$$

To prove that $u$ is a solution to Problem $(P)$ amounts to proving that under some assumptions, the mapping $\varphi\left(y_{f}^{\sigma}, \cdot\right)$ has one unique fixed point $u^{*}$ in the ball $B(0, m)$ of $L^{2}(0, T ; \mathcal{U})$.

In fact, apart from (A1)-(A5), we assume the following:

(A6) $\exists g_{2} \in L^{q}(0, T)$ :

$$
\left\|C \chi_{\sigma}^{*} \chi_{\sigma} S_{f}(t)\right\|_{\mathcal{L}(Z, V)} \leq g_{2}(t), \quad \forall t \in[0, T] .
$$

(A7) $T<\frac{1}{\left(K^{*}\left\|g_{1}\right\|_{q}+\beta K^{*}\left\|g_{2}\right\|_{q}\right)^{p}}$

(A8) $\left.\exists \lambda^{\prime} \in\right] 0,1\left[: \alpha\left\|z_{0}\right\|_{X}+\gamma\|f\|_{\mathcal{F}}\right.$

$$
\in] 0, \frac{\lambda^{\prime}}{T^{\frac{1}{p}}}\left(1-T^{\frac{1}{p}}\left(k\left\|g_{1}\right\|_{q}+\beta k\left\|g_{2}\right\|_{q}\right)\right)[.
$$

Theorem 2. If Assumptions (A1)-(A8) are satisfied, then there exist $d$ and $\rho=\rho(d)>0$ such that for all $y_{f}^{\sigma} \in$ $B(0, \rho) \subset V$, where $y_{f}^{\sigma}$ is given by (22), the mapping $\varphi\left(y_{f}^{\sigma}, \cdot\right)$ has a unique fixed point $u^{*}$ in $B(0, m)$.

Proof. We have

$$
\lim _{\left(\theta_{1} ; \theta_{2}\right) \rightarrow(0,0)} K\left(\theta_{1} ; \theta_{2}\right)=0 .
$$

Then

$\forall \varepsilon>0, \exists d \in] 0,1\left[\sup _{\left\|\left(\theta_{1} ; \theta_{2}\right)\right\|<d} K\left(\theta_{1} ; \theta_{2}\right)<\varepsilon\right.$.

In particular, for

$$
\varepsilon=\left[T^{\frac{1}{p}}\left(\left\|g_{1}\right\|_{q}+\beta\left\|g_{2}\right\|_{q}\right)\right]^{-1}
$$

we obtain

$$
\left.c_{2}=T^{\frac{1}{p}}\left(\left\|g_{1}\right\|_{q}+\beta\left\|g_{2}\right\|_{q}\right) K \in\right] 0,1[.
$$


Let us prove that $\varphi\left(y_{f}^{\sigma}, B(0, m)\right) \subset B(0, m)$ for $y_{f}^{\sigma} \in V$, where $m$ and $y_{f}^{\sigma}$ are respectively given by 17] and (22). Indeed, for $u \in B(0, m)$ we have

$$
\begin{aligned}
\left\|\varphi\left(y_{f}^{\sigma}, u\right)\right\|_{L^{2}(0, T ; \mathcal{U})} & =\left\|y_{f}^{\sigma}-C \chi_{\sigma}^{*} \chi_{\sigma} \widehat{H}_{f, T} h_{1}(u)\right\|_{V} \\
& \leq\left\|y_{f}^{\sigma}\right\|_{V}+\left\|C \chi_{\sigma}^{*} \chi_{\sigma} \widehat{H}_{f, T} h_{1}(u)\right\|_{V} .
\end{aligned}
$$

Then by (A2) and (A6) we obtain

$$
\begin{aligned}
& \left\|\varphi\left(y_{f}^{\sigma}, u\right)\right\|_{L^{2}(0, T ; \mathcal{U})} \\
& \quad \leq\left\|y_{f}^{\sigma}\right\|_{V}+\left\|g_{2}\right\|_{q} K\left(\left\|h_{1}(u)\right\|_{p} ; 0\right)\left\|h_{1}(u)\right\|_{p} .
\end{aligned}
$$

Using (9) and the fact that $u \in B(0, m)$ implies $h_{1}(u) \in B(0, m)$, we obtain

$$
\left\|\varphi\left(y_{f}^{\sigma}, u\right)\right\|_{L^{2}(0, T ; \mathcal{U})} \leq\left\|y_{f}^{\sigma}\right\|_{V}+k\left\|g_{2}\right\|_{q} d .
$$

Thus the condition

$$
u \in B(0, m) \Rightarrow \varphi\left(y_{f}^{\sigma}, u\right) \in B(0, m)
$$

is satisfied if

$$
\left\|y_{f}^{\sigma}\right\|_{V} \leq m-k\left\|g_{2}\right\|_{q} d .
$$

From 177 we obtain $\left\|y_{f}^{\sigma}\right\|_{V} \leq \rho$ with

$$
\begin{aligned}
\rho= & \frac{1}{\beta}\left[\left(\frac{1-T^{\frac{1}{p}}\left(k\left\|g_{1}\right\|_{q}+\beta k\left\|g_{2}\right\|_{q}\right)}{T^{\frac{1}{p}}}\right) d\right. \\
& \left.-\alpha\left\|z_{0}\right\|_{X}-\gamma\|f\|_{\mathcal{F}}\right] .
\end{aligned}
$$

As a consequence of (A7) we have

$$
1-T^{\frac{1}{p}}\left(k\left\|g_{1}\right\|_{q}+\beta k\left\|g_{2}\right\|_{q}\right)>0 .
$$

In addition, using (A8) with $\lambda^{\prime}=d$ we obtain $\rho>$ 0 . Then, if $y_{f}^{\sigma} \in B(0, \rho)$, we get $\varphi\left(y_{f}^{\sigma}, B(0, m)\right) \subset$ $B(0, m)$.

Moreover, for $y_{f}^{\sigma} \in B(0, \rho)$ the mapping $\varphi\left(y_{f}^{\sigma}, \cdot\right)$ is a contraction in $B(0, m)$. Indeed, for $u, v \in B(0, m)$ we have

$$
\begin{aligned}
\varphi\left(y_{f}^{\sigma}, u\right) & -\varphi\left(y_{f}^{\sigma}, v\right) \\
= & L\left(C \chi_{\sigma}^{*} \chi_{\sigma} \widehat{H}_{f, T} h_{1} u-C \chi_{\sigma}^{*} \chi_{\sigma} \widehat{H}_{f, T} h_{1} v\right) .
\end{aligned}
$$

Using (24) we get

$$
\begin{aligned}
\left\|\varphi\left(y_{f}^{\sigma}, u\right)-\varphi\left(y_{f}^{\sigma}, v\right)\right\|_{L^{2}(0, T ; \mathcal{U})} \\
=\left\|C \chi_{\sigma}^{*} \chi_{\sigma} \widehat{H}_{f, T} h_{1} u-C \chi_{\sigma}^{*} \chi_{\sigma} \widehat{H}_{f, T} h_{1} v\right\|_{V} \\
=\| \int_{0}^{T} C \chi_{\sigma}^{*} \chi_{\sigma} S_{f}(T-s)\left[N_{f} h_{1}(u)(s)\right. \\
\left.\quad-N_{f} h_{1}(v)(s)\right] \mathrm{d} s \|_{V} .
\end{aligned}
$$

From $(A 2)$ and $(A 6)$ it follows that

$$
\begin{aligned}
\left\|\varphi\left(y_{f}^{\sigma}, u\right)-\varphi\left(y_{f}^{\sigma}, v\right)\right\|_{L^{2}(0, T ; \mathcal{U})} \\
\quad \leq\left\|g_{2}\right\|_{q} K\left\|h_{1}(u)-h_{1}(v)\right\|_{p} .
\end{aligned}
$$

Since the mapping $h_{1}$ is Lipschitzian with the coefficient $T^{\frac{1}{p}} \beta / 1-c_{1}$ (by the point (b) of Theorem 1 , we have

$$
\left\|\varphi\left(y_{f}^{\sigma}, u\right)-\varphi\left(y_{f}^{\sigma}, v\right)\right\|_{L^{2}(0, T ; \mathcal{U})} \leq c_{3}\|u-v\|_{L^{2}(0, T ; \mathcal{U})}
$$

with

$$
c_{3}=\frac{T^{\frac{1}{p}} \beta\left\|g_{2}\right\|_{q} K}{1-c_{1}},
$$

which means that $\varphi\left(y_{f}^{\sigma}, \cdot\right)$ is a contraction on $B(0, m)$ as $0<c_{3}<1$. Indeed, from the constants $c_{1}, c_{2}$ and $c_{3}$, given respectively by (16), 27) and 29, we obtain

$$
1-c_{3}=\frac{1-c_{1}-T^{\frac{1}{p}} \beta\left\|g_{2}\right\|_{q} K}{1-c_{1}}=\frac{1-c_{2}}{1-c_{1}} .
$$

Since $\left.c_{1}, c_{2} \in\right] 0,1\left[\right.$ and $c_{1}<c_{2}$, we have $0<c_{3}<1$.

Finally, for $y_{f}^{\sigma} \in B(0, \rho)$ the mapping $\varphi\left(y_{f}^{\sigma}, \cdot\right)$ has a unique fixed point $u^{*}$ in $B(0, m)$ which is a solution to Problem $(P)$.

For Theorem 2, we have a result ensuring the convergence of a sequence of controls associated with 25) to the solution $u^{*}$ of Problem $(P)$.

Proposition 2. If the assumptions of Theorem 2 are satisfied and if $y_{f}^{\sigma} \in V$ is such that $\left\|y_{f}^{\sigma}\right\|_{V} \leq \rho$, then the sequence defined by

$$
\left\{\begin{aligned}
u_{0} & =0 \\
u_{n+1} & =\varphi\left(y_{f}^{\sigma}, u_{n}\right)=L\left(y_{f}^{\sigma}-C \chi_{\sigma}^{*} \chi_{\sigma} \widehat{H}_{f, T} h_{1}\left(u_{n}\right)\right)
\end{aligned}\right.
$$

converges to $u^{*}$ in $L^{2}(0, T, \mathcal{U})$ and satisfies

$$
\left\|u_{n+1}-u_{n}\right\|_{L^{2}(0, T ; \mathcal{U})} \leq c_{3}^{n}\left\|u_{1}\right\| .
$$

The proof of this proposition is deduced from Theorem 2 and the properties of the fixed point, where $c_{3}$ is a constant given by (29).

Thus if we consider

$$
\begin{gathered}
z_{f, u_{n}}^{n}=h_{1}\left(u_{n}\right), \quad y_{f, u_{n}}^{\sigma, n}(T)=C \chi_{\sigma}^{*} \chi_{\sigma} z_{f, u_{n}}^{n}(T), \\
r_{n+1}=y_{f}^{\sigma}-C \chi_{\sigma}^{*} \chi_{\sigma} \widehat{H}_{f, T} z_{f, u_{n}}^{n},
\end{gathered}
$$

then using the output function $(E),(20)$ and (22) we have

$$
\begin{aligned}
y_{f, u_{n}}^{\sigma, n}(T)= & C \chi_{\sigma}^{*} \chi_{\sigma} S_{f}(T) z_{0}+C \chi_{\sigma}^{*} \chi_{\sigma} \widehat{H}_{f, T} z_{f, u_{n}}^{n} \\
& +C \chi_{\sigma}^{*} \chi_{\sigma} \widetilde{H}_{f, T} f+C \chi_{\sigma}^{*} \chi_{\sigma} H_{f, T} u_{n} \\
= & y_{f}^{\sigma}+C \chi_{\sigma}^{*} \chi_{\sigma} S_{f}(T) z_{0} \\
& +C \chi_{\sigma}^{*} \chi_{\sigma} \widehat{H}_{f, T} z_{f, u_{n}}^{n}+C \chi_{\sigma}^{*} \chi_{\sigma} \widetilde{H}_{f, T} f \\
& +C \chi_{\sigma}^{*} \chi_{\sigma} H_{f, T} u_{n}-y_{f}^{\sigma} \\
= & y_{0,0}^{\sigma}(T)-C \chi_{\sigma}^{*} \chi_{\sigma} f-r_{n+1}+r_{n} .
\end{aligned}
$$

Then

$$
r_{n+1}=r_{n}+y_{0,0}^{\sigma}(T)-y_{f, u_{n}}^{\sigma, n}(T)-C \chi_{\sigma}^{*} \chi_{\sigma} f .
$$

On the other hand, it is easy to see that

$$
\left\|y_{f, u_{n}}^{\sigma, n}(T)-y_{0,0}^{\sigma}(T)-C \chi_{\sigma}^{*} \chi_{\sigma} f\right\|_{V} \leq c_{3}^{n}\left\|u_{1}\right\| .
$$




\section{Numerical approach}

In this section, we outline a numerical approach which allows us to compute the solution to Problem $(P)$. An illustrative example is developed using two choices of inputs and outputs. We recall the following definitions (El Jai et al., 1986):

Definition 3. Let $D_{1}$ and $D_{2}$ be closed subsets of $\Omega$, and $g \in L^{2}\left(D_{1}\right), h \in L^{2}\left(D_{2}\right)$. We have the following:

(i) A zone actuator is a couple $\left(D_{1}, g\right)$, where

- $D_{1}$ is the space support of the actuator,

- $g$ defines the spatial distribution of the actuator.

(ii) A zone sensor is a couple $\left(D_{2}, h\right)$, where

- $D_{2}$ is the space support of the sensor,

- $h$ defines the spatial distribution of the sensor.

In the pointwise case, we have similar definitions. Indeed, a pointwise actuator (respectively a sensor) is a couple $\left(b, \delta_{b}\right)$ (resp., $\left.\left(c, \delta_{c}\right)\right)$, where $b, c \in \Omega$ are the locations and $\delta$ is the Dirac mass. The notion of a regional efficient actuator is introduced in (Afifi et al., 2002) and is related to the concept of regional remediability as defined below.

Definition 4. The actuator $\left(D_{1}, g\right)$ is said to be $\sigma$ efficient if the system $(\widetilde{S})$ is weakly $\sigma$-remediable when it is excited by the actuator $\left(D_{1}, g\right)$.

Remark 8. All the explored results of this paper assume that $B$ and $C$ belong to $\mathcal{L}(U ; Z)$ and $\mathcal{L}(Z ; Y)$, respectively. In the pointwise case, additional hypotheses must be considered.

Using the above remark in what follows, we are going to limit ourselves to the zonal case. Then, before exploring an illustrative example, we provide an algorithm resulting from the above results.

5.1. Algorithm. We recall the problem considered as stated above:

$$
(P)\left\{\begin{array}{l}
\text { Find } u \in L^{2}(0, T ; \mathcal{U}) \text { such that } \\
y_{f, u}^{\sigma}(T)=y_{0,0}^{\sigma}(T)-C \chi_{\sigma}^{*} \chi_{\sigma} f .
\end{array}\right.
$$

Based on Proposition 2 we can develop an algorithm which allows us to determine a sequence of controls which converges to the solution $u^{*}$ of $(P)$. The output $(E)$ is given by

$$
\begin{aligned}
y_{f, u}^{\sigma}(t)= & C \chi_{\sigma}^{*} \chi_{\sigma} S_{f}(t) z_{0}+C \chi_{\sigma}^{*} \chi_{\sigma} \widehat{H}_{f, t} z_{f, u} \\
& +C \chi_{\sigma}^{*} \chi_{\sigma} \widetilde{H}_{f, t} f+C \chi_{\sigma}^{*} \chi_{\sigma} H_{f, t} u .
\end{aligned}
$$

\section{Algorithm 1.}

1. Data: $\Omega, \sigma, T, z_{0}, \omega, f, A, N, B, C, \varepsilon$.

2. Compute $y_{0,0}^{\sigma}$ : the output $(E)$ when $f=0$ and $u=0$ (an autonomous case).

3. Compute $y_{f, 0}^{\sigma}$ : the output $(E)$ when $f \neq 0$ and $u=0$ (a disturbed case).

4. Let $r_{0}=0$ and $r_{1}=y_{0,0}^{\sigma}(T)-y_{f, 0}^{\sigma}(T)-C \chi_{\sigma}^{*} \chi_{\sigma} f$.

5. Compute $u_{n}=L r_{n}$ where $L$ is given by (23).

6. Compute $y_{f, u_{n}}^{\sigma, n}$ : the output $(E)$.

7. If $\left\|u_{n}-u_{n-1}\right\|_{L^{2}(0, T ; \mathcal{U})} \leq \varepsilon$ then stop.

Otherwise, consider $r_{n+1}$ given by (31) and go to Step 5.

Above, $L$ is the pseudoinverse of the linear operator $C \chi_{\sigma}^{*} \chi_{\sigma} C H_{f, T}$ which can be computed using (14) and (23),

$$
\begin{aligned}
(L r)(t)= & {\left[\int_{0}^{T} C \chi_{\sigma}^{*} \chi_{\sigma} S_{f}(\tau) B B^{*} S_{f}^{*}(\tau) \chi_{\sigma}^{*} \chi_{\sigma} C^{*} \mathrm{~d} \tau\right]^{-1} } \\
& \times B^{*} S_{f}^{*}(T-t) \chi_{\sigma}^{*} \chi_{\sigma} C^{*} r
\end{aligned}
$$

The main difficulty in the implementation of the above algorithm is the computation of the pseudo inverse and the solution of the partial differential equation (Step 6 of the algorithm).

In the next section we outline an example by means of the above algorithm.

5.2. A one-dimensional transport system. Let $\Omega=$ ] $0, \pi / 4[$ and $I=] 0, T[$ with $T=2$. Consider the nonlinear distributed system defined in $\Omega \times I$ and governed by the following state equation:

$$
\left\{\begin{array}{l}
\dot{\tilde{z}}(x, t)=-c \frac{\partial \widetilde{z}}{\partial x}+\widetilde{z}^{2}(x, t)+B u(t), \\
\quad 0<x<\pi / 4,0<t<2, \\
\widetilde{z}(x, 0)=z_{0}(x)+f(x), \quad 0<x<\pi / 4,
\end{array}\right.
$$

with $\widetilde{z} \in L^{2}(0, T, Z), Z=L^{2}(\Omega)$ and $c>0$. Set $c=$ $3 \pi / 32$.

Let $\omega=] 0, \pi / 16[(\omega \subset \Omega)$ be the geometrical support of the disturbance $f$. Consider the following initial data and disturbance given respectively by

$$
\begin{gathered}
z_{0}(x)=0, \quad \forall x \in \Omega, \\
f(x)= \begin{cases}-\cos (x) & \text { if } x \in \omega, \\
0 & \text { otherwise. }\end{cases}
\end{gathered}
$$


Let $\sigma$ be a fixed subregion in $\Omega$ which does not necessarily satisfy $\sigma \cap \omega \neq \emptyset$. The system (34) is assumed to be augmented by the output function

$$
(\widetilde{E}) \quad \widetilde{y}^{\sigma}(t)=C \chi_{\sigma}^{*} \chi_{\sigma} \widetilde{z}(t) .
$$

The remediability problem associated with this example will be treated by considering various forms of $\sigma$, actuators (the choice of $B$ ) and sensors (the choice of $C$ ).

Case 1: $\sigma=[3 \pi / 16,7 \pi / 32]$.

Consider $B u(t)=g(x) u(t)$, where

$$
g(x)= \begin{cases}\exp \left(-x^{2}\right) & \text { if } 3 \pi / 16 \leq x \leq 7 \pi / 32 \\ 0 & \text { otherwise }\end{cases}
$$

and

$$
C \chi_{\sigma}^{*} \chi_{\sigma} \widetilde{z}(t)=\int_{3 \pi / 16}^{7 \pi / 32} h(x) \widetilde{z}(x, t) \mathrm{d} x,
$$

where

$$
h(x)= \begin{cases}\exp (x) & \text { if } 3 \pi / 16 \leq x \leq 7 \pi / 32 \\ 0 & \text { otherwise }\end{cases}
$$

The system (34) is a particular case of (1) with

$$
F \widetilde{z}=-c \frac{\partial \widetilde{z}}{\partial x}+\widetilde{z}^{2}
$$

and

$$
B u(t)=g(x) u(t) .
$$

The assumptions on $F$ are satisfied because we have $F(0)=0$ and $F$ is of class $C^{1}$.

Let $z(x, t)=\widetilde{z}(x, t)-f(x)$. The system 34 becomes

$$
\left\{\begin{aligned}
\dot{z}(x, t)= & -c\left(\frac{\partial z}{\partial x}(x, t)+\frac{\partial f}{\partial x}\right) \\
& +(z(x, t)+f(x))^{2}+g(x) u(t) \\
z(x, 0)= & 0 .
\end{aligned}\right.
$$

Linearizing $F$ around $f$, the system $(35)$ can be rewritten in the form

$$
\left\{\begin{aligned}
\dot{z}(x, t)= & \left(-c \frac{\partial}{\partial x}+2 f(x)\right) z(x, t)+z^{2}(x, t) \\
& +\left(f^{2}(x)-c \frac{\partial f}{\partial x}(x)\right)+g(x) u(t), \\
z(x, 0)= & 0 .
\end{aligned}\right.
$$

Consider the linear operator

$$
A_{f}=-c \frac{\partial}{\partial x}+2 f, \mathcal{D}\left(A_{f}\right) \longrightarrow Z
$$

with the domain

$$
\mathcal{D}\left(A_{f}\right)=\left\{z \in Z:-c \frac{\partial z}{\partial x}+2 f z \in Z\right\}
$$

densely included in $Z$. $A_{f}$ generates a strongly continuous semigroup given by

$\left(S_{f}(t) z\right)(x)= \begin{cases}\exp [2 f(x) t] z(x-c t), & x>c t, \\ 0, & 0<x \leq c t\end{cases}$

which satisfies (A1) with $g_{1}(t)=\exp (-2 \cos (\pi / 16) t)$ $\left(g_{1} \in L^{2}(0,2)\right)$.

The nonlinear operator $N_{f}: z \in X=L^{4}(\Omega) \longmapsto$ $z^{2} \in Z=L^{2}(\Omega)$ is well defined in a Banach space $X$ and satisfies (A2) because $N_{f}(0)=0$ and, with the Hölder inequality, we obtain $\forall t \in[0,2]$ :

$$
\begin{aligned}
& \left\|N_{f} z_{1}(t)-N_{f} z_{2}(t)\right\|_{L^{2}(\Omega)} \\
& =\left\|z_{1}^{2}(t)-z_{2}^{2}(t)\right\|_{L^{2}(\Omega)} \\
& =\left\|\left(z_{1}+z_{2}\right)(t)\left(z_{1}-z_{2}\right)(t)\right\|_{L^{2}(\Omega)} \\
& \quad \leq\left(\left\|z_{1}(t)\right\|_{X}+\left\|z_{2}(t)\right\|_{X}\right)\left\|z_{1}(t)-z_{2}(t)\right\|_{X} \\
& \quad \leq \sqrt{2}\left[\left\|z_{1}(t)\right\|_{X}^{2}+\left\|z_{2}(t)\right\|_{X}^{2}\right]^{\frac{1}{2}}\left\|z_{1}(t)-z_{2}(t)\right\|_{X} \\
& \quad \leq K\left(\left\|z_{1}\right\|_{2} ;\left\|z_{2}\right\|_{2}\right)\left\|z_{1}(t)-z_{2}(t)\right\|_{X},
\end{aligned}
$$

where

$$
K\left(\left\|z_{1}\right\|_{2} ;\left\|z_{2}\right\|_{2}\right)=\max _{t}\left[\left\|z_{1}(t)\right\|_{X}^{2}+\left\|z_{2}(t)\right\|_{X}^{2}\right]^{\frac{1}{2}} .
$$

It is clear that $K: \mathbb{R}_{+} \times \mathbb{R}_{+} \longrightarrow \mathbb{R}_{+}$satisfies

$$
\lim _{\left(\theta_{1} ; \theta_{2}\right) \rightarrow(0,0)} K\left(\theta_{1} ; \theta_{2}\right)=0 .
$$

Let $d=0.8$ and

$$
\begin{gathered}
T_{1}=\frac{1}{\left(K^{*}\left\|g_{1}\right\|_{2}\right)^{2}}, \quad f_{1}=\alpha\left\|z_{0}\right\|_{X}+\gamma\|f\|_{X}, \\
\lambda_{1}=\frac{d}{T^{\frac{1}{2}}}\left(1-T^{\frac{1}{2}} k\left\|g_{1}\right\|_{2}\right) .
\end{gathered}
$$

Then, using numerical computations, we get Table 1. This shows that (A3)-(A5) are satisfied since the constants $\alpha$, $\beta, \gamma$ are positive, and the conditions $T=2<T_{1}$ (respectively, $f_{1}<\lambda_{1}$ ) hold. Moreover, the constants $c_{1}$ and $m$, given by (16) and (17), satisfy $\left.c_{1} \in\right] 0,1[$ and $m>0$. Consequently, from Theorem 1 the system (36) has a unique solution given for all $t \in[0,2]$ by

$$
\begin{aligned}
z_{f, u}(t)= & \int_{0}^{t} S_{f}(t-s) z_{f, u}^{2}(s) \mathrm{d} s \\
& +\int_{0}^{t} S_{f}(t-s)\left(f^{2}-c \frac{\partial f}{\partial x}\right) \mathrm{d} s \\
& +\int_{0}^{t} S_{f}(t-s) g u(s) \mathrm{d} s .
\end{aligned}
$$


Table 1. Constants of Theorem 1

\begin{tabular}{|c|c|c|c|c|c|c|c|}
\hline$\alpha$ & $\beta$ & $\gamma$ & $T_{1}$ & $f_{1}$ & $\lambda_{1}$ & $m$ & $c_{1}$ \\
\hline 1 & 0.1053 & 0.4453 & 6.9772 & 0.2945 & 0.3285 & 0.3229 & 0.5354 \\
\hline
\end{tabular}

To solve the regional remediability problem $(P)$ (and then $(\widetilde{P})$ ), consider the disturbed controlled system (36) together with the output $(E)$ given by

(E) $y^{\sigma}(t)=C \chi_{\sigma}^{*} \chi_{\sigma} z(t)=\int_{3 \pi / 16}^{7 \pi / 32} h(x) z(x, t) \mathrm{d} x$.

We have to find a control $u$ such that

$\int_{0}^{2} \int_{3 \pi / 16}^{7 \pi / 32} h(x) S_{f}(2-s) g(x) u(s) \mathrm{d} x \mathrm{~d} s$

$+\int_{0}^{2} \int_{3 \pi / 16}^{7 \pi / 32} h(x) S_{f}(2-s) z_{f, u}^{2}(x, s) \mathrm{d} x \mathrm{~d} s$

$+\int_{0}^{2} \int_{3 \pi / 16}^{7 \pi / 32} h(x) S_{f}(2-s)\left(f^{2}-c \frac{\partial f}{\partial x}\right)(x) \mathrm{d} x \mathrm{~d} s$

$+\int_{3 \pi / 16}^{7 \pi / 32} h(x) f(x) \mathrm{d} x$

$-\int_{0}^{2} \int_{3 \pi / 16}^{7 \pi / 32} h(x) S_{0}(2-s) z_{0,0}^{2}(x, s) \mathrm{d} x \mathrm{~d} s=0$.

It is clear that (A6) holds with

$$
\left.g_{2}(t)=\|h\|_{L^{2}(\sigma)} \exp (-2 \cos (\pi / 16) t) \quad \forall t \in\right] 0,2[.
$$

Similarly, if we set

$$
\begin{aligned}
T_{2} & =\frac{1}{\left(K^{*}\left\|g_{1}\right\|_{2}+\beta K^{*}\left\|g_{2}\right\|_{2}\right)^{2}}, \\
\lambda_{2} & =\frac{d\left(1-T^{\frac{1}{2}}\left(k\left\|g_{1}\right\|_{2}+\beta k\left\|g_{2}\right\|_{2}\right)\right)}{T^{\frac{1}{2}}},
\end{aligned}
$$

then we obtain the results given in Table 2. Hence we

\section{Table 2. Constants of Theorem 2}

\begin{tabular}{|c|c|c|c|c|c|}
\hline$T_{2}$ & $f_{1}$ & $\lambda_{2}$ & $\rho$ & $c_{2}$ & $c_{3}$ \\
\hline 6.1804 & 0.2945 & 0.3049 & 0.0981 & 0.5689 & 0.0720 \\
\hline
\end{tabular}

deduce that (A7) and (A8) are also satisfied because we have $T<T_{2}$ and $f_{1}<\lambda_{2}$. Thus the constants $c_{2}, \rho$ and $c_{3}$, given respectively by (27), (28) and 29), satisfy $\rho>0$ and $\left.c_{2}, c_{3} \in\right] 0,1[$. Then from Theorem 2 the problem (37) has a unique solution $u$.

5.3. Simulation results. When the system (34) is not disturbed $(f=0)$ and it is autonomous $(u=0)$, the solution in this case is given by

$$
\left.z_{0,0}(x, t)=0, \quad(x, t) \in\right] 0, \pi / 4[\times] 0,2[.
$$

When the autonomous system $(u=0)$ is disturbed by $f$, the solution becomes

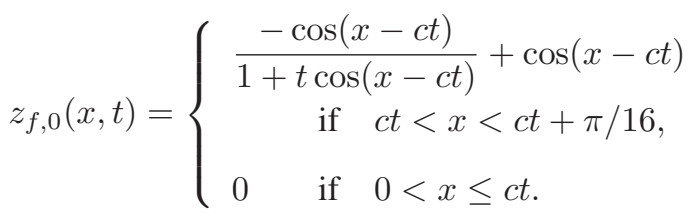

As for the solution to (34) given by $\tilde{z}_{f, u}(x, t)=$ $z_{f, u}(x, t)+f(x)$, Fig. 3 shows the states $\widetilde{z}_{0,0}$ and $\widetilde{z}_{f, 0}$. In Fig. 3(b) we have the state of the autonomous system

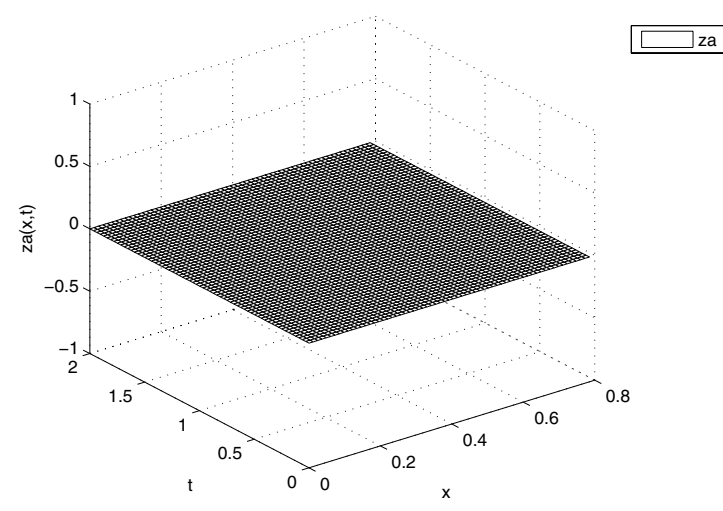

(a)

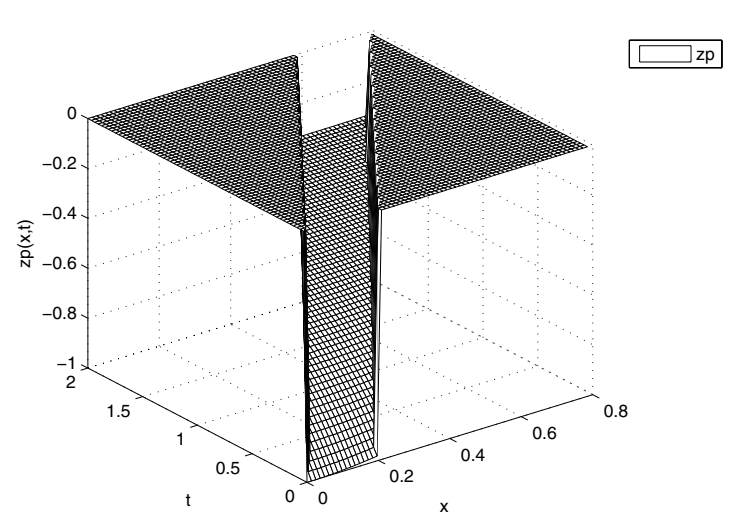

(b)

Fig. 3. States in the autonomous case (a) and in the disturbed uncontrolled one (b).

$\widetilde{z}_{0,0}$ and that of the disturbed system $\widetilde{z}_{f, 0}$. The disturbance $f$ effect is important. 
Consider the property $\mathcal{P}$ given by $\mathcal{P} \widetilde{z}_{f, 0}(x, t) \Leftrightarrow$ $\widetilde{z}_{f, 0}(x, t) \neq \widetilde{z}_{0,0}(x, t)$, and write

$$
w_{f, 0, t}=\left\{x \in \Omega: \mathcal{P} \widetilde{z}_{f, 0}(x, t)\right\} .
$$

As $\widetilde{z}_{0,0}(x, t)=0, \forall(x, t) \in \Omega \times I$, and $\widetilde{z}_{f, 0}(x, t) \neq 0$, $\forall x \in] c t, c t+\pi / 16[, t \neq 0$, we have

$$
\left.w_{f, 0, t}=\right] c t, c t+\pi / 16[\text {. }
$$

Consequently, we have the result given in Fig. 4. Namely, the disturbance $f$ is spreadable and the zone $\sigma$ considered is $f$-vulnerable.

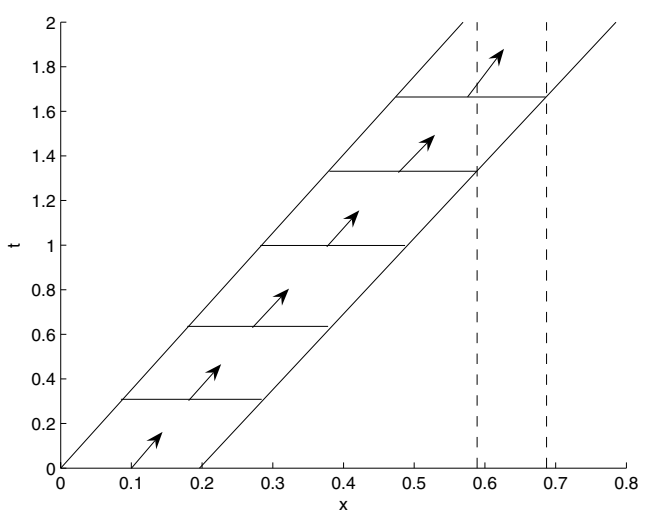

Fig. 4. Spreadability of the disturbance $f$ and vulnerability of $\sigma \simeq[0.59,0.69]$.

Consider times $t_{1}$ and $t_{2}$ given by

$$
\begin{gathered}
t_{1}=\inf \left\{t \in I: \stackrel{\circ}{\sigma} \cap w_{f, 0, t} \neq \emptyset\right\}, \\
t_{2}=\inf \left\{t \in\left[t_{1}, T\right]: \bigcup_{s \in\left[t_{1}, t_{2}\right]}\left(\stackrel{\circ}{\sigma} \cap w_{f, 0, s}\right)\right. \\
\left.=\bigcup_{s \in\left[t_{1}, T\right]}\left(\stackrel{\circ}{\sigma} \cap w_{f, 0, s}\right)\right\} .
\end{gathered}
$$

This two instants signify respectively the first time when the zone $\sigma$ is reached and the first one when the bigger part of $\sigma$ is reached, which can be $\sigma$. In this case (see Fig. 4), by using expressions of $c, w_{f, 0, t}$ and $\sigma$, we have $t_{1}=4 / 3$ and $t_{2}=5 / 3$.

Thus the spreadable disturbance begins to reach $\sigma$ at time $t_{1}$. It affects all the subregion at time $t_{2}$ and the entire $\sigma$ is reached during the time interval $\left[t_{2}, T\right]$. Then the solution to 37 produces a control which compensates in $\sigma$ the effect of the spreadable disturbance $f$.

Thus, when we apply Algorithm 1 with a space step $\Delta x=0.01$, a time step $\Delta t=0.03$, and for $\varepsilon=10^{-4}$, we obtain the evolution shown in Figs. 5 and 6.

In Fig. 5, we present the state of the disturbed controlled system $\widetilde{z}_{f, u}$ which shows that in $\sigma$ it is very similar to the autonomous state $\widetilde{z}_{0,0}$ at time $T$ as $n$ increases. This

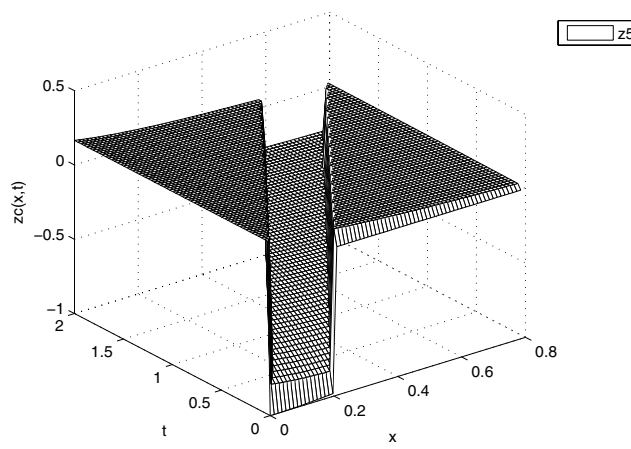

(a)

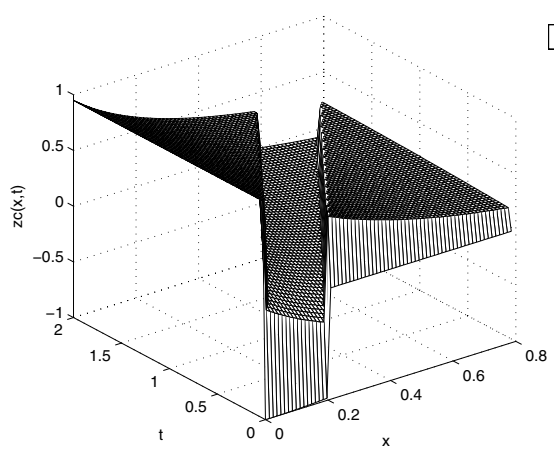

(b)

Fig. 5. Controlled case: the states for $n=5$ (a) and $n=20$ (b).

is also shown in Fig. 6, where the states $\widetilde{z}_{f, u}(\cdot, T)$ and the outputs $\widetilde{y}_{f, u}$ are very close to $\widetilde{z}_{0,0}(\cdot, T)$ and $\widetilde{y}_{0,0}$, respectively, at the final time $T=2$.

In fact, these results show that the control $u$ of the remediability problem (37) is regionally efficient since it neutralizes the spatial disturbance space effect $f$ in the subregion $\sigma$.

We can also see that the evolution of states in $\sigma$ (respectively the associated outputs) in the controlled case is due to the choice of the spatial distribution of the actuator $g$ (resp. the sensor $h$ ) given in Fig. 7 .

The sequence of controls converges to $u$ (Fig. 8), and they are increasing because the disturbed state (or the associated output) is located in the lower part of that of the normal system. Figure 8 b) shows the error dependence on the iteration number $k$. If we consider the margin error $E(k)$ between the outputs $\widetilde{y}_{f, u}(T)$ and $\widetilde{y}_{0,0}(T)$, then we notice that $E(k)$ decreases to 0 as $k \rightarrow \infty$, showing the convergence of the algorithm where the Lipschitz constant is equal to $c_{3}=0.0720$. We also computed the cost of such remediability in $\sigma$ and obtained $\|u\|_{L^{2}(0, T)}=0.2992$.

Remark 9. As has been mentioned, the transport velocity $c$ is positive. Consequently, the disturbance is spreadable. 


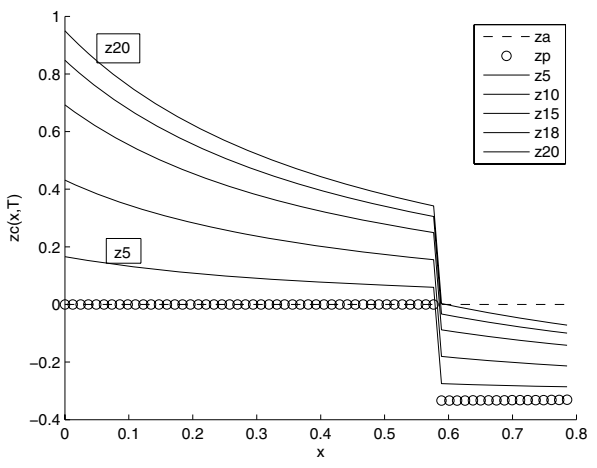

(a)

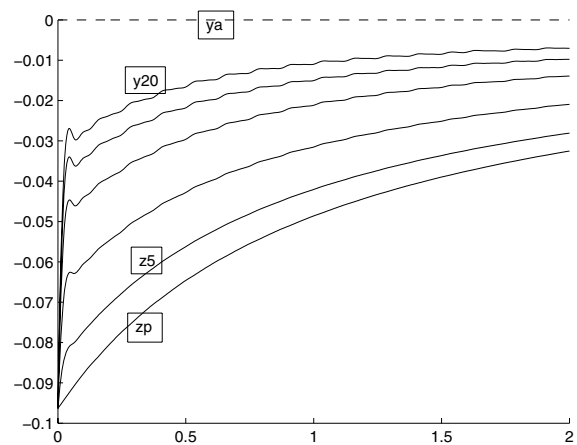

(b)

Fig. 6. States of the autonomous, disturbed and controlled cases at the final time $T=2$ (a) and the associated outputs (b) for $n=5,10,15,18$ and 20 .

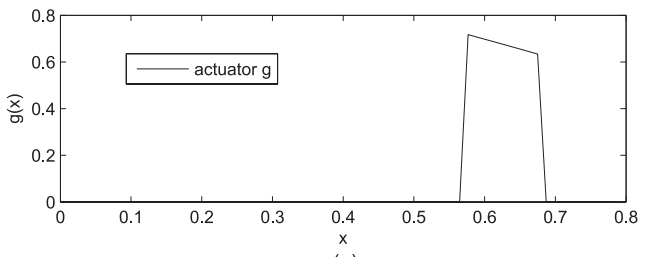

(a)

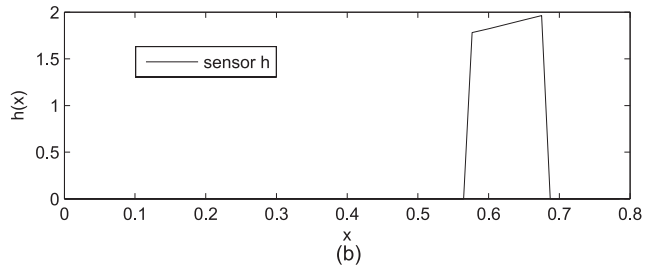

Fig. 7. Spatial distributions of the actuator $g(a)$ and the sensor $h(\mathrm{~b})$

In the case when $c \leq 0$, the disturbance is resorbable (or stationary for $c=0$ ), the subregion $\sigma$ considered is not vulnerable and then no remediability is needed.

An interesting situation is when $c=0$ and $\sigma=\omega=$ $\Omega$. At the initial time the geometrical support of the disturbance $f$ becomes the whole domain $\Omega$.

Case 2: $\omega=\sigma=\Omega=] 0, \pi / 4[, B=I$ and $C=I$.

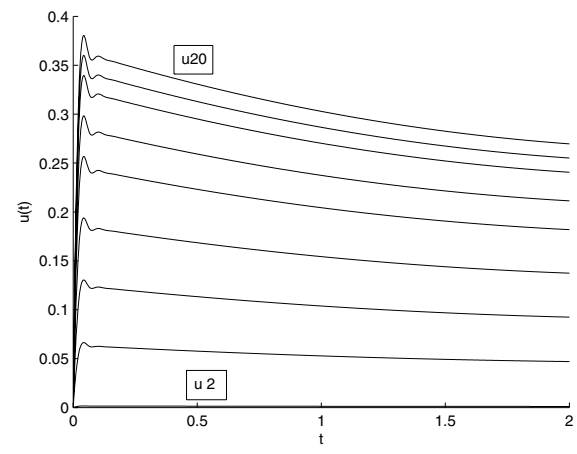

(a)

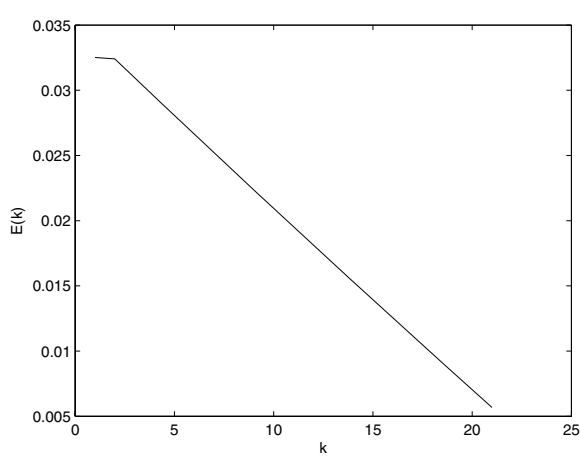

(b)

Fig. 8. Sequence of controls (a) and the error between the outputs $y_{0,0}(T)$ and $y_{f, u}(T)(b)$.

In much the same way as in the first case, it is easy to show that all the assumptions of Theorems 1 and 2 are satisfied. Then we have to find a control $u$ satisfying

$$
\begin{aligned}
\int_{0}^{2} & \int_{0}^{\pi / 4} e^{2 f(x)(2-s)} u(s) \mathrm{d} x \mathrm{~d} s \\
& +\int_{0}^{2} \int_{0}^{\pi / 4} e^{2 f(x)(2-s)} z_{f, u}^{2}(x, s) \mathrm{d} x \mathrm{~d} s \\
+ & \int_{0}^{2} \int_{0}^{\pi / 4} e^{2 f(x)(2-s)} f^{2}(x) \mathrm{d} x \mathrm{~d} s \\
+ & \int_{0}^{\pi / 4} f(x) \mathrm{d} x-\int_{0}^{2} \int_{0}^{\pi / 4} z_{0,0}^{2}(x, s) \mathrm{d} x \mathrm{~d} s=0 .
\end{aligned}
$$

With the same algorithm we obtain the evolution shown in Figs. 99

In Fig. (9), we give the state of the autonomous system $\widetilde{z}_{0,0}$ (a) and that of the disturbed system $\widetilde{z}_{f, 0}(\mathrm{~b})$. The disturbance $f$ is globally defined in the whole domain $\Omega=\sigma$. Consequently, this domain is affected by the effect of the disturbance.

In this case the subregion $\sigma$ is the entire geometrical domain $\Omega$, the actuator is distributed on $\Omega$ with, $B=I$, and the output is given by means of one distributed sensor, $C=I$. From Fig. 10 it is seen that the state of the 


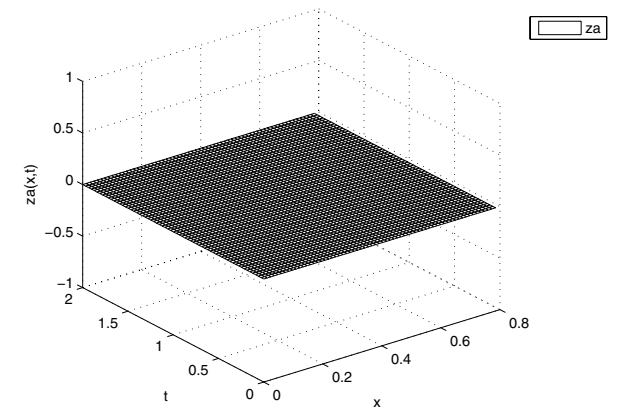

(a)

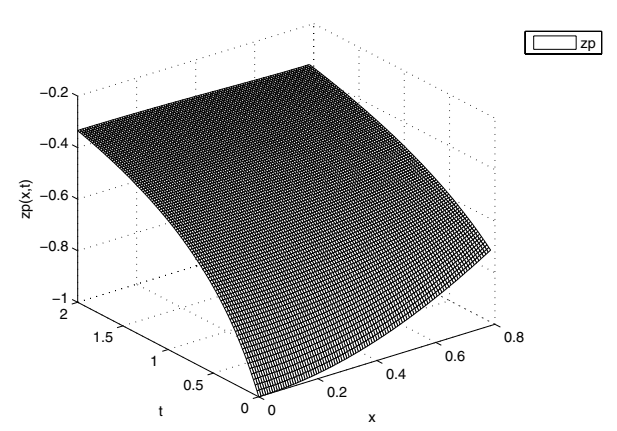

(b)

Fig. 9. State of the autonomous case (a) and the disturbed case (b).

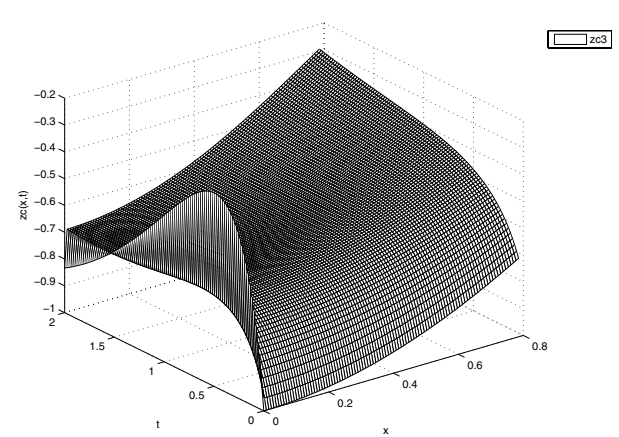

(a)

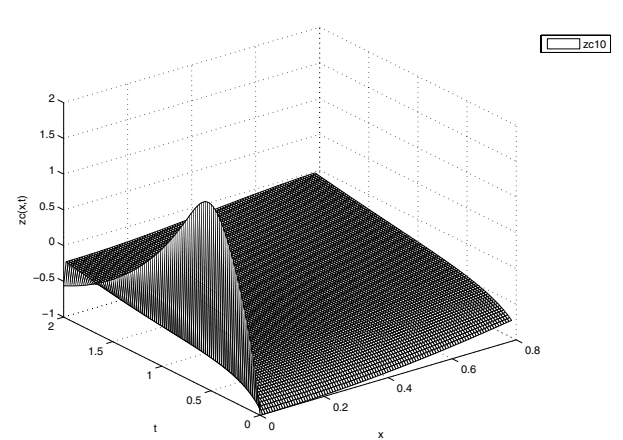

(b)

Fig. 10. Controlled case: the states for $n=3$, and $n=10$.

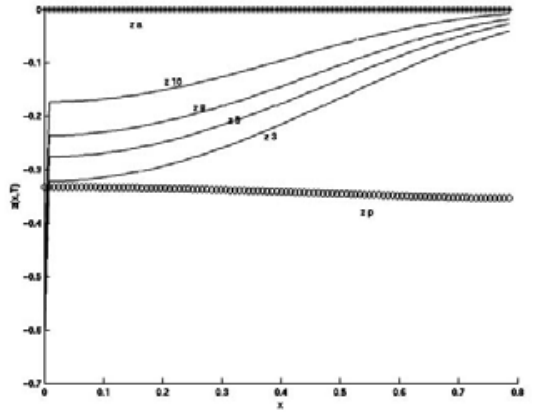

(a)

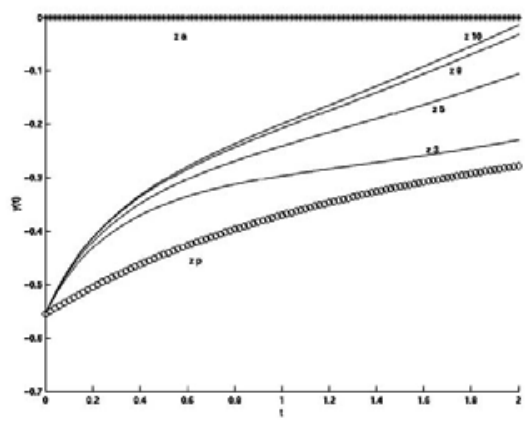

(b)

Fig. 11. States of the autonomous, disturbed and controlled cases at the final time $T=2$ (a) and the associated outputs (b) for $n=3,5,8$ and 10 .

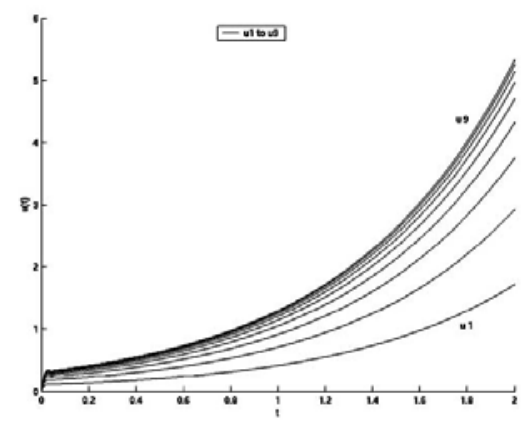

(a)

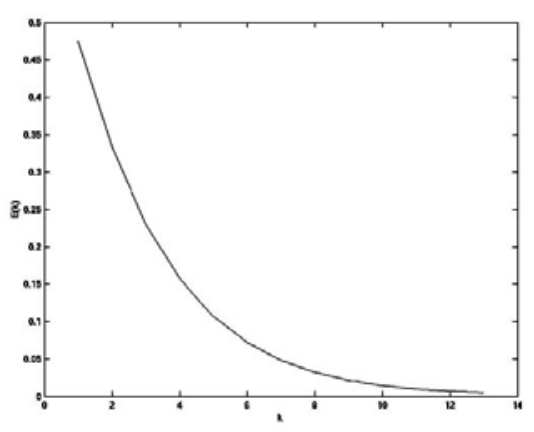

(b)

Fig. 12. Sequence of controls for $n=2, \ldots, 10$ (a) and the error between the outputs $y_{0,0}(T)$ and $y_{f, u}(T),(b)$. 
disturbed controlled system $\widetilde{z}_{f, u}$ is very similar to the autonomous state $\widetilde{z}_{0,0}$ at time $T$ as $n$ increases. Also, from Fig. 11 we observe that the states $\widetilde{z}_{f, u}(\cdot, T)$ and the outputs $\widetilde{y}_{f, u}$ are respectively gradually near $\widetilde{z}_{0,0}(\cdot, T)$ and $\widetilde{y}_{0,0}$ at the final time $T=2$.

In Fig. 12 a) we see that the sequence of controls converges to the solution $u$ of the global remediability problem (38). This shows that the control $u$ is efficient since it neutralized the spatial disturbance effect $f$ in the whole domain $\Omega$. The cost of such remediability is $\|u\|_{L^{2}(0, T)}=5.7303$.

Similarly to the regional case, the increasing control sequence is due to the fact that the disturbed state (or the associated output) is located at the lower part of that of the normal system, which is a consequence of the homogeneous boundary condition in $x=0$. In Fig. 10(b) we presented the error dependence on the iteration number $k$. More precisely, we consider the error margin $E(k)$ between the outputs $\widetilde{y}_{f, u}(T)$ and $\widetilde{y}_{0,0}(T)$. One can notice that the error margin $E(k)$ decreases to 0 as $k \rightarrow \infty$, showing the convergence of the algorithm.

\section{Conclusion}

In this work we studied the problem of regional remediability for a class of nonlinear distributed systems. The space disturbance occurs in the initial condition. This problem was solved combining pseudo inverse techniques and the fixed-point theorem. An example was developed numerically to illustrate the various steps. The discussed transformation of the system in the neighborhood of the disturbance enables us to solve the remediability problem and, in addition, allows the connection between the concepts of remediability and spreadability through that of vulnerability. Based on this approach, it will be very interesting to consider the protector control problem for nonlinear distributed parameter systems as it was introduced for linear systems by Qaraai et al. (Qaraai et al., 2006; Qaraai et al., 2008). This will be published in a future work.

\section{Acknowledgements}

This work is supported by the NATO Collaborative Grant No. 982891 and the AUF Project No. 6313PS559 as well as by Académie Hassan II des Sciences et Techniques in Morocco.

\section{References}

Afifi L., El Jai A. and Zerrik E.(2008). Analyse régionale des systèmes linéaires à paramétres distribués, Press Universitaires de Perpignan, In press.

Afifi L., A. Chafiai and A. El Jai (2002). Regionally efficient and strategic actuators, International Journal of Systems Science 33 (4): 1-12.
Bernoussi A. (2007). Spreadability and vulnerability of distributed parameter systems, International Journal of Systems Science 38(4): 305-317.

Bernoussi A. and A. El Jai, (2000). New approach of spreadability, Journal of Mathematical and Computer Modelling 31(13): 93-109.

Bernoussi A., A. El Jai and A. J. Pritchard (2001). Spreadability and evolving interfaces, International Journal of Systems Science 32(10): 1217-1232.

Bernoussi A. and M. Amharref (2003). Etalabilité-vulnérabilité, Annals of University of Craiova, Mathematics and Computer Science Series 30: 53-62. ISSN 1223-6934.

Cholewa J. W. and T. Dłotko (2000). Global Attractors in Abstract Parabolic Problems, Cambridge University Press, London Mathematical Society.

Christofides P. D. (1998). Robust control of parbolic PDE Systems, Chemical Engineering Science 53: 2949-2965.

Christofides P.D. and P. Daoutidis (1998). Robust control of hyperbolic PDE systems, Chemical Engineering Science 53: 85-105.

Christofides P.D. (2001). Nonlinear and Robust Control of PDE Systems: Methods and Applications to Transport-Reaction Processes, Springer, Berkhäuser.

Curtain R. F. and A.J. Pritchard (1978). Infinite Dimensional Linear Systems Theory, Springer, Berlin-Heidelberg, New York, pp. 297.

Dautray R. F. and J. L. Lions (1984). Analyse mathématique et calcul numérique pour les sciences et les techniques, Vol. 3, Masson, Paris.

Doubova A., E. Fernández-Cara, M. González-Burgos and E. Zuazua (2006). On the controllability of parabolic systems with a nonlinear term involving the state and the gradient, SIAM Journal on Control and Optimization 45(4): 13991446.

El Jai A. and A. J. Pritchard (1986). Capteurs et actionneurs dans l'analyse des systems distribués, Masson, Paris.

El Jai A. and K. Kassara (1994). Spreadable distributed systems. Mathematical and Computer Modelling 20(1): 47-64.

El Jai A., M.C. Simon, E. Zerrik and A.J. Pritchard (1995). Regional controllability of distributed systems, International Journal of Control 62(6): 1351-1365.

El Jai A. (2002). Analyse régionale des systémes distribués, ESAIM: Control, Optimisation and Calculus of Variations 8: 663-692.

El Jai A. (2004). Eléments d'Analyse et de Contrôle des Systémes, Presses Universitaires de Perpignan.

Henry D. (1981). Geometric Theory of Semilinear Parabolic Systems, Springer, New York.

Kassara K. and A. El Jai (1983). Algorithmes pour la commande d'une classe de systems à paramètres répartis non linéaires, Revue Marocaine d'Automatique, d'Informatique et de Traitement de Signal 1(1): 3-24.

Pazy A. (1983). Semigroups of Linear Operators and Applications to Partial Differential Equations, Springer-Verlag, New York. 
Qaraai Y., A. Bernoussi and A. El Jai (2006). Contrôle protecteur et remédiabilité, Proceedings of the 3rd International Congress on Scientific Advances in Civil and Industrial Engineering, Algeciras, Spain, pp. 107-114.

Qaraai Y., A. Bernoussi and A. El Jai (2008). Protector control of distributed parameter systems, International Journal of Systems Science, submitted.

Received: 12 April 2007

Revised: 29 October 2007 
